\title{
Charge states of strongly correlated 3d oxides: from typical insulator to unconventional electron-hole Bose liquid
}

\author{
A.S. Moskvin ${ }^{1}$ \\ ${ }^{1}$ Department of Theoretical Physics, Ural State University, 620083, Ekaterinburg, Russia
}

\begin{abstract}
We develop a model approach to describe charge fluctuations and different charge phases in strongly correlated 3d oxides. As a generic model system one considers that of centers each with three possible valence states $M^{0, \pm}$ described in frames of $S=1$ pseudo-spin (isospin) formalism by an effective anisotropic non-Heisenberg Hamiltonian which includes two types of single particle correlated hopping and the two-particle hopping. We show that the coherent states provide the optimal way both to a correct mean-field approximation and respective continuous models to describe the pseudo-spin system. Simple uniform mean-field phases include an insulating monovalent $M^{0}$ phase, mixed-valence binary (disproportionated) $M^{ \pm}$-phase, and mixed-valence ternary ("underdisproportionated") $M^{0, \pm}$-phase. We consider two first phases in more details focusing on the problem of electron/hole states and different types of excitons in $M^{0}$-phase and formation of electronhole Bose liquid in $M^{ \pm}$-phase. Pseudo-spin formalism provides a useful framework for revealing and describing different topological charge fluctuations, in particular, like domain walls or bubble domains in antiferromagnets. Electron-lattice polarization effects are shown to be crucial for the stabilization of either phase. All the insulating systems such as $M^{0}$-phase may be subdivided to two classes: stable and unstable ones with regard to the formation of self-trapped charge transfer (CT) excitons. The latter systems appear to be unstable with regard to the formation of CT exciton clusters, or droplets of the electron-hole Bose liquid. The model approach suggested is believed to be applied to describe a physics of strongly correlated oxides such as cuprates, manganites, bismuthates, and other systems with charge transfer excitonic instability and/or mixed valence. We shortly discuss an unconventional scenario of the essential physics of cuprates and manganites that implies their instability with regard to the self-trapping of charge transfer excitons and the formation of electron-hole Bose liquid.
\end{abstract}

\section{INTRODUCTION}

The discovery of the high- $T_{c}$ superconductivity in doped cuprates, $\frac{1}{\underline{1}}$ observation of many unconventional properties in doped manganites with their colossal magnetoresistance, bismuthates with high- $T_{c}$ 's, nickellates and many other oxides ${ }^{2}$ shows that we deal with a manifestation of novel strongly correlated states with a local charge instability, mixed valence, "metal-dielectric" duality, strong coupling of different (charge, spin, orbital, structural) degrees of freedom and non-Landau behaviour of quasiparticles. All this has generated a flurry of ideas, models and scenarios of the puzzling transport phenomena and stimulated the intensive studies of various correlation effects and charge transfer (CT) phenomena in strongly correlated systems derived in either way from insulators unstable with regard to the CT fluctuations. Conventional approach to hotly debated strongly correlated $3 \mathrm{~d}$ oxides such as cuprates, manganites, and many other similar systems implies making use of a Hubbard model with famous Hamiltonian

$$
\hat{H}=-\sum_{<i, j>, \sigma} t(i j) \hat{c}_{i \sigma}^{\dagger} \hat{c}_{j \sigma}+U \sum_{i, \sigma \sigma^{\prime}} n_{i \sigma} n_{i \sigma^{\prime}}
$$

with competing contributions of kinetic and potential terms. Here $\hat{c}_{i \sigma}^{\dagger} / \hat{c}_{j \sigma}$ are creation/annihilation operators for low-lying antibonding 3d-O 2 p hybridized orbitals. The two-center charge transfer integral $t(i j)$ is often associated with d-d transfer. Mott-Hubbard insulator is believed to arise from a potentially metallic half-filled band as a result of the Coulomb blockade of electron tunnelling $(U \gg t)$ to neighboring sites $\frac{3}{\underline{3}}$

Despite intense effort, the behavior of strongly correlated 3d oxides remain poorly understood and we are still far from a comprehensive understanding of the underlying physics. Moreover, it seems that there are missing qualitative aspects of the problem beyond the simple Hubbard scenario that so far escaped the identification and the recognition. Firstly it concerns strong electronlattice polarization effects which may be subdivided into electron-lattice interaction itself,, 4.5 and a contribution of an electronic background that is electronic subsystem which is not incorporated into effective Hubbard model Hamiltonian 6.7 These effects are of great importance for the ground state electronic and crystalline structure, and can seriously modify the doping response of $3 \mathrm{~d}$ oxide up to the crucial change of the seemingly natural ground state. This question has not received the attention it deserves. It should be emphasized that traditional Fröhlich approach to the electron-lattice coupling implies the description of linear effects whereas the charge fluctuations in the insulator do imply strongly nonlinear electronlattice coupling with the predominance of polarization and relaxation effects, and another energy scale.

Electron-lattice effects may be directly incorporated into effective Hubbard model. Assuming the coupling with the local displacement (configuration) coordinate $Q$ in the effective potential energy we arrive at a generalized Peierls-Hubbard model $\stackrel{8}{*}$ From the other hand, the 
taking account of similar effects in the kinetic energy results in a generalized Su-Schrieffer-Heeger (SSH) model ${ }^{9}$ The correlation effect of an electronic background was shown ${ }^{6.7}$ to be of primary importance for atomic systems with filled or almost filled electron shells. Namely such a situation is realized in oxides with $\mathrm{O}^{2-}\left(2 \mathrm{p}^{6}\right)$ oxygen ions. In particular, the effect results in a correlated character of a charge transfer that seems to be one of the main features for $3 \mathrm{~d}$ oxides.

Many strongly correlated 3d oxides reveal anomalous sensitivity to a small nonisovalent substitution. For example, only $2 \% \mathrm{Sr}^{2+}$ substituted for $\mathrm{La}^{3+}$ in $\mathrm{La}_{2} \mathrm{CuO}_{4}$ result in a dramatical suppression of long-range copper antiferromagnetism, while it is suppressed with isovalent $\mathrm{Cu}^{2+}$ substitution by $\mathrm{Zn}^{2+}$ at a much higher concentration close to the site dilution percolating threshold. Simultaneously, the transport properties of $\mathrm{La}_{2-x} \mathrm{Sr}_{x} \mathrm{CuO}_{4}$ system reveal unconventional insulator-metal duality starting from very low dopant level $\stackrel{10}{\underline{10}}$ Most likely, all this points to a charge phase instability intrinsic for parent 214 system which somehow evolves with nonisovalent substitution due to a well developed charge potential inhomogeneity and/or hole doping effect. The problem seems to be closely related with the hidden multistability intrinsic to each solid. $\stackrel{811}{ }$ If the ground state of a solid is pseudo-degenerate, being composed of true and false ground states with each structural and electronic orders different from others, one might call it multi-stable. Below we focus ourselves on a charge degree of freedom and charge (in)stability, rather than orbital or spin degrees of freedom. As an illuminating example of such a material with conceptually simple but actually false ground state Toyozawa 11 suggests to address the Wolfram's red, a quasi-one dimensional material of which the skeleton chain consists of alternate array: $\left(\mathrm{Cl}^{-}-\mathrm{Pt}^{3+}-\right)^{2 n}$ with simple (and seemingly metallic), but a false ground state. The real ground state is an insulator with a complicated structure of doubled period: $\left(\mathrm{Cl}^{-}-\mathrm{Pt}^{4+}-\mathrm{Cl}^{-}-\mathrm{Pt}^{2+}\right)^{n}$, which can be reached from the former through the Peierls transition with the charge density wave of large amplitude, or disproportionation like reaction. This transition can be considered as the condensation of self-decomposed self-trapped excitons spontaneously generated on all unit cells.

In this connection it is worth noting the text-book example of $\mathrm{BaBiO}_{3}$ system where we unexpectedly deal with the disproportionated $\mathrm{Ba}^{3+}+\mathrm{Ba}^{5+}$ ground state instead of the conventional lattice of $\mathrm{Ba}^{4+}$ cations. ${ }^{12}$ The bismuthate situation can be viewed also as a result of a condensation of CT excitons, in other words, the spontaneous generation of self-trapped CT excitons in the ground state with a proper transformation of lattice parameters. At present, a CT instability with regard to disproportionation is believed to be a rather typical property for a number of perovskite $3 \mathrm{~d}$ transitionmetal oxides such as $\mathrm{SrFeO}_{3}, \mathrm{LaCuO}_{3}, \mathrm{RNiO}_{3}{ }^{13}$, moreover, in solid state chemistry one consider tens of dispro-

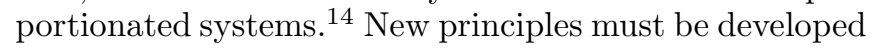

to treat such charge or CT unstable systems with their dramatical non-Fermi-liquid behavior. In particular, we have to change the current paradigm of the metal-toinsulator (MI) transition to that of an insulator-to-metal (IM) phase transition. These two approaches imply essentially different starting points: the former starts from a rather simple metallic-like scenario with inclusion of correlation effects, while the latter does from strongly correlated atomic-like scenario with the inclusion of a charge transfer. Electron-lattice polarization effects accompanying the charge transfer appear to be of primary importance to stabilize either phase state. One should emphasize that the theoretical description of such systems is one of the challenging problems in solid state physics.

Hereafter, we develop a model approach to describe different charge fluctuations and charge phases in strongly correlated 3d oxides with main focus on the correlated $\mathrm{CT}$ effects. As an illustrative model system we address a simple mixed-valence system with three possible stable nondegenerate valent states of a cation-anionic cluster, hereafter $M: M^{0}, M^{ \pm}$, forming the charge (isospin) triplet. The $M^{0}$ valent state is associated with the conceptually simple one like $\mathrm{CuO}_{4}^{6-}$ in insulating copper oxides $\left(\mathrm{CuO}, \mathrm{La}_{2} \mathrm{CuO}_{4}, \mathrm{YBa}_{2} \mathrm{Cu}_{3} \mathrm{O}_{6}, \mathrm{Sr}_{2} \mathrm{CuO}_{2} \mathrm{Cl}_{2}, \ldots\right)$ or $\mathrm{MnO}_{6}^{9-}$ in manganite $\mathrm{LaMnO}_{3}$ or $\mathrm{BiO}_{6}^{9-}$ in bismuthates. It is worth noting that such a model is a most relevant to describe different cuprates where novel concepts should compete with a traditional Hubbard model approach in a hole representation implying the vacuum state formed by $M^{-}\left(\mathrm{CuO}_{4}^{5-}\right)$ centers, and some concentration of holes. That is why overall the paper we refer the insulating cuprates to illustrate the main concepts of the approach developed. Our mathematics is based on the $S=1$ pseudo-spin formalism (see e.g. review article Ref 15) to be the effective tool for the description of the essential physics both of insulators unstable with regard to the CT fluctuations and related mixed-valence systems. Such an approach provides the universal framework for a unified description of these systems as possible phase states of a certain parent multi-stable system. In addition, we may make use of powerful methods developed in the physics of spin systems. The model system of $M^{0, \pm}$ centers is described in frames of $S=1$ pseudo-spin formalism by an effective anisotropic non-Heisenberg Hamiltonian which includes two types of correlated two-center hopping:

$$
M^{0}+M^{0} \leftrightarrow M^{ \pm}+M^{\mp} \text { and } M^{ \pm}+M^{0} \leftrightarrow M^{0}+M^{ \pm},
$$

respectively. It should be noted that we neglect all the intra-center transition, including anion-cation $\mathrm{O} 2 \mathrm{p}-3 \mathrm{~d}$ charge transfer.

Our main goal is to describe different charge phases of the model system and a scenario of evolution of visibly typical insulator to unconventional electron-hole Bose liquid which reveals many unexpected properties, including superconductivity. The paper is organized as follows: In Sec.II we address a metal-oxide cluster model, different mechanisms of correlation effects, and the ef- 
fects of electron-lattice polarization. In Sec.III we introduce the $S=1$ pseudo-spin formalism to describe the model mixed-valence systems. The effective pseudospin Hamiltonian and possible mean-field phase states of the mixed-valence systems are discussed in Sec.IV. In Sec.V we analyse an eh-representation of different excitations in a monovalent $M^{0}$ phase, discuss a CT instability, and nucleation of electroh-hole $(\mathrm{EH})$ droplets. Electronhole Bose liquid is discussed in Sec.VI. Some topological skyrmion-like charge fluctuations in the model mixed valence system are described in Sec.VII. Implications for cuprates and manganites are discussed in Sec.VIII.

\section{METAL-OXIDE CLUSTERS AND CORRELATION EFFECTS}

The electronic states in strongly correlated $3 \mathrm{~d}$ oxides manifest both significant correlations and dispersional features. The dilemma posed by such a combination is the overwhelming number of configurations which must be considered in treating strong correlations in a truly bulk system. One strategy to deal with this dilemma is to restrict oneself to small 3d-metal-oxygen clusters, creating model Hamiltonians whose spectra may reasonably well represent the energy and dispersion of the important excitations of the full problem. Indeed, such clusters as $\mathrm{CuO}_{4}$ in quasi-2D cuprates, $\mathrm{MnO}_{6}$ in manganite perovskites are basic elements of crystalline and electronic structure. Despite a number of principal shortcomings, including the boundary conditions, the breaking of local symmetry of boundary atoms, sharing of common anions for $n n$ clusters etc., the embedded molecular cluster method provides both, a clear physical picture of the complex electronic structure and the energy spectrum, as well as the possibility of quantitative modelling. Eskes et $a l^{16}$, as well as Ghijsen et al. ${ }^{17}$ have shown that in a certain sense the cluster calculations might provide a better description of the overall electronic structure of insulating $3 \mathrm{~d}$ oxides than band-structure calculations. In particular, they allow to take better into account different correlation effects.

Below, in the Section we discuss some aspects of electronic structure, energy spectrum, and correlation effects for an illustrative example of $\mathrm{CuO}_{4}$ clusters embedded into an insulating cuprate.

\section{A. Electronic structure of copper-oxygen clusters}

Beginning from $5 \mathrm{Cu} 3 d$ and 12 O $2 p$ atomic orbitals for $\mathrm{CuO}_{4}$ cluster with $D_{4 h}$ symmetry, it is easy to form 17 symmetrized $a_{1 g}, a_{2 g}, b_{1 g}, b_{2 g}, e_{g}$ (gerade=even) and $a_{2 u}, b_{2 u}, e_{u}(\sigma), e_{u}(\pi)$ (ungerade=odd) orbitals. The even $\mathrm{Cu} 3 d \quad a_{1 g}\left(3 d_{z^{2}}\right), b_{1 g}\left(3 d_{x^{2}-y^{2}}\right), b_{2 g}\left(3 d_{x y}\right), e_{g}\left(3 d_{x z}, 3 d_{y z}\right)$ orbitals hybridize, due to strong $\mathrm{Cu} 3 d$-O $2 p$ covalency, with even $\mathrm{O} 2 p$-orbitals of the same symmetry, thus forming appropriate bonding $\gamma^{b}$ and antibonding $\gamma^{a}$

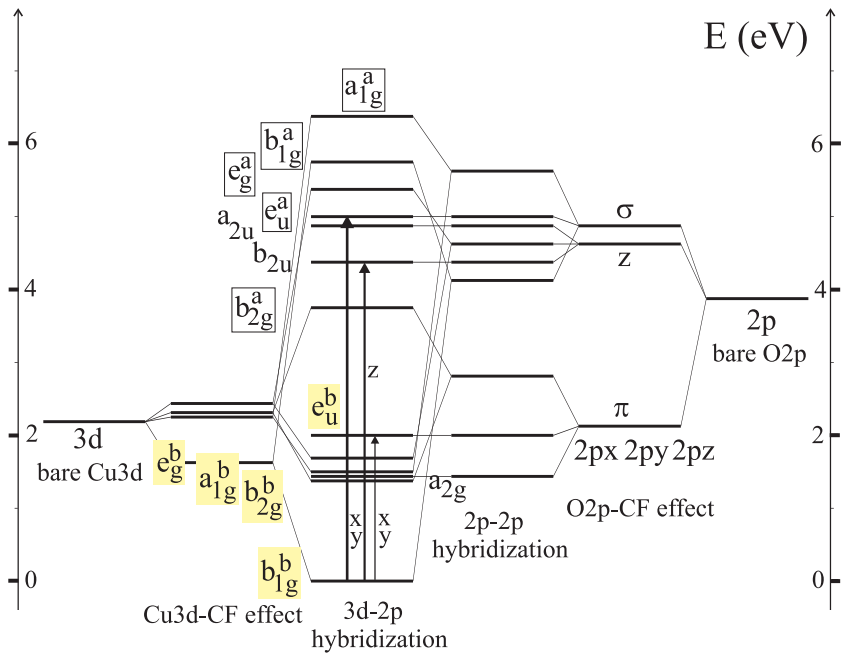

FIG. 1: Model single-hole energy spectra for a $\mathrm{CuO}_{4}$ plaquette with parameters relevant for a number of insulating cuprates.

states. Among the odd orbitals only $e_{u}(\sigma)$ and $e_{u}(\pi)$ hybridize due to nearest neighbor $p p$ overlap and transfer thus forming appropriate bonding $e_{u}^{b}$ and antibonding $e_{u}^{a}$ purely oxygen states. The purely oxygen $a_{2 g}, a_{2 u}, b_{2 u}$ orbitals are nonbonding. All "planar" O $2 p$ orbitals in accordance with the orientation of lobes could be classified as $\sigma\left(a_{1 g}, b_{1 g}, e_{u}(\sigma)\right)$ or $\pi\left(a_{2 g}, b_{2 g}, e_{u}(\pi)\right)$ orbitals, respectively.

Bonding and antibonding molecular orbitals in hole representation can be presented, for example, as follows

$$
\begin{aligned}
& \left|b_{1 g}^{b}\right\rangle=\cos \alpha_{b_{1 g}}\left|b_{1 g}(3 d)\right\rangle+\sin \alpha_{b_{1 g}}\left|b_{1 g}(2 p)\right\rangle, \\
& \left|b_{1 g}^{a}\right\rangle=\sin \alpha_{b_{1 g}}\left|b_{1 g}(3 d)\right\rangle-\cos \alpha_{b_{1 g}}\left|b_{1 g}(2 p)\right\rangle
\end{aligned}
$$

where $b_{1 g}(3 d)=3 d_{x^{2}-y^{2}}$ and $\left|b_{1 g}(2 p)\right\rangle$ is a superposition of four $\mathrm{O} 2 \mathrm{p}$ orbitals with $b_{1 g}$ symmetry.

Fig. 1 presents a single-hole energy spectrum for a $\mathrm{CuO}_{4}$ plaquette embedded into an insulating cuprate like $\mathrm{Sr}_{2} \mathrm{CuO}_{2} \mathrm{Cl}_{2}$ calculated with a reasonable set of parameters. 18 For illustration we show also a step-bystep formation of the cluster energy levels from the bare $\mathrm{Cu} 3 d$ and $\mathrm{O} 2 p$ levels with the successive inclusion of crystalline field (CF) effects, $\mathrm{O} 2 p-\mathrm{O} 2 p$, and $\mathrm{Cu} 3 d-\mathrm{O}$ $2 p$ covalency. It is worth noting that strong $\mathrm{Cu} 3 \mathrm{~d}-\mathrm{O}$ $2 \mathrm{p}$ overlap and covalency result in a dramatic difference between $\mathrm{CuO}_{4}^{6-}$ center and $\mathrm{Cu}^{2+}$ cation as its naive analogue.

\section{Two-hole configurations for the $\mathrm{CuO}_{4}^{5-}$ center}

Starting from $\mathrm{CuO}_{4}^{6-}$ center as a realization of $M^{0}$ center we arrive at $\mathrm{CuO}_{4}^{5-}$ and $\mathrm{CuO}_{4}^{7-}$ centers as realizations of hole $M^{+}$and electron $M^{-}$centers, respectively. The electron $\mathrm{CuO}_{4}^{7-}$ center has a filled set of $\mathrm{Cu} 3 \mathrm{~d}$ and $\mathrm{O}$ 
$2 \mathrm{p}$ orbitals, and is often addressed to be a generalization of $\mathrm{Cu}^{1+}$ ion. The hole $\mathrm{CuO}_{4}^{5-}$ center has actually twohole configuration with the lowest Zhang-Rice (ZR) spinsinglet ${ }^{1} A_{1 g}$ state ${ }^{19}$ formed by the interaction of three "covalent" configurations: $\left(b_{1 g}^{a}\right),\left(b_{1 g}^{b}\right)$, and $\left(b_{1 g}^{a} b_{1 g}^{b}\right)$, respectively, or three "purely ionic" two-hole configurations $\left|d^{2}\right\rangle,|p d\rangle$, and $\left|p^{2}\right\rangle$. Here, $|d\rangle=\left|d_{x^{2}-y^{2}}\right\rangle$ and $|p\rangle=\left|p_{b_{1 g}}\right\rangle$ are the non-hybridized $\mathrm{Cu} 3 d_{x^{2}-y^{2}}$ and $\mathrm{O} 2 p_{\sigma}$ orbitals, respectively, with bare energies $\epsilon_{d}$ and $\epsilon_{p}$.

Let us present a simple example of the calculation of the two-hole spectrum in the ZR-singlet sector. The matrix of the full effective Hamiltonian within the bare purely ionic basis set has a rather simple form

$$
\hat{H}=\left(\begin{array}{ccc}
2 \epsilon_{d}+U_{d} & t & 0 \\
t & \epsilon_{d}+\epsilon_{p}+V_{p d} & t \\
0 & t & 2 \epsilon_{p}+U_{p}^{*}
\end{array}\right),
$$

where the effective Coulomb parameter for purely oxygen configuration incorporates both the intra-atomic parameter $U_{p}$ and the oxygen-oxygen coupling to the first and second nearest neighbors, respectively

$$
U_{p}^{*}=U_{p}+\frac{1}{4} V_{p p}^{(1)}+\frac{1}{8} V_{p p}^{(2)}
$$

and the following condition holds:

$$
U_{d}>U_{p}>V_{p d}
$$

For reasonable values of parameters (in $\mathrm{eV}): U_{d}=8.5$, $U_{p}=4.0, V_{p d}=1.2, \epsilon_{d}=0, \epsilon_{p}=3.0, t=t_{p d}=1.3(\mathrm{see}$ Ref. 18) we obtain for the ZR-singlet energy $E_{Z R}=3.6$, and its wave function

$$
\left|\Phi_{1}^{(2)}\right\rangle=\left|b_{1 g}^{2} ; p d\right\rangle=0.25\left|d^{2}\right\rangle-0.95|p d\rangle+0.19\left|p^{2}\right\rangle,
$$

where $|p d\rangle=\frac{1}{\sqrt{2}}(|p(1) d(2)\rangle+|p(2) d(1)\rangle)$. It reflects the well-known result that the ZR-singlet represents a twohole configuration with one predominantly $\mathrm{Cu} 3 d$ and one predominantly $\mathrm{O} 2 p$ hole. It is worth noting that the hole $\mathrm{CuO} \mathrm{O}_{4}^{5-}$ center sometimes one naively associate with $\mathrm{Cu}^{3+}$ ion, however, such a conclusion is a rather far from reality. Indeed, this center is the mixed valence one, as the $C u$ valence resonates between +2 and +3 .

The two excited states with energies $E_{Z R}+5.2$ and $E_{Z R}+6.7 \mathrm{eV}$ are described by the wave functions

$$
\begin{gathered}
\left|\Phi_{2}^{(2)}\right\rangle=\left|b_{1 g}^{2} ; d d\right\rangle=-0.95\left|d^{2}\right\rangle-0.21|p d\rangle+0.22\left|p^{2}\right\rangle \\
\left|\Phi_{3}^{(2)}\right\rangle=\left|b_{1 g}^{2} ; p p\right\rangle=0.17\left|d^{2}\right\rangle+0.24|p d\rangle+0.96\left|p^{2}\right\rangle
\end{gathered}
$$

respectively. Given the ZR-singlet energy one may calculate the minimal energy for $M^{0}+M^{0} \rightarrow M^{ \pm}+M^{\mp}$ charge transfer:

$$
\Delta_{C T}=E_{Z R}-2 E_{b_{1 g}}=(3.6+0.5) \mathrm{eV}=4.1 \mathrm{eV},
$$

where the stabilization energy for the bonding $b_{1 g}^{b}$ state is simply calculated from matrix (3) at $U_{d}=U_{p}^{*}=V_{p d}=0$.
It should be emphasized that this quantity plays a particular role as the minimal charge transfer energy which specifies the charge transfer gap. In the general case it is defined as follows:

$$
\Delta_{C T}=E_{N+1}+E_{N-1}-2 E_{N},
$$

or as the energy required to remove a hole from one region of the crystal and add it to another region beyond the range of excitonic correlations. The exact diagonalization studies for a series of clusters with different size ${ }^{20.21}$ show that $\Delta_{C T}$ strongly diminishes with cluster size from $\approx$ $4 \mathrm{eV}$ for small clusters to $\approx 2.5 \mathrm{eV}$ as extrapolated value for large clusters.

Our simple calculation points to a significant role of correlation effect. Indeed, the inter-configurational coupling due to Coulomb repulsion results in a visible deviation of the two-hole ground state wave function from the predictions of simple model of noninteracting particles

$$
\begin{gathered}
\left|\left(b_{1 g}^{b}\right)^{2}\right\rangle=(-0.8|d(1)\rangle+0.6|p(1)\rangle)(-0.8|d(2)\rangle+0.6|p(2)\rangle) \\
=0.64\left|d^{2}\right\rangle-0.68|p d\rangle+0.36\left|p^{2}\right\rangle .
\end{gathered}
$$

In particular, it could give rise to a strong renormalization of the hole transfer integrals.

\section{B. Electron-lattice polarization effects}

\section{Correlation effects of electronic background}

The correlation problem becomes of primary importance for atoms/ions near Coulomb instability when the one-electron gluing cannot get over the destructive effect of the electron-electron repulsion. Such a situation seems to realize in oxides where Hirsch et $a l^{6}$ have proposed an instability of $\mathrm{O}^{2-}\left(2 \mathrm{p}^{6}\right)$ electronic background. The main suggestion in their theory of "anionic metal" concerns the occurrence of the non-rigid degenerate structure for a closed electron shell such as $\mathrm{O}^{2-}\left(2 \mathrm{p}^{6}\right)$ with the internal purely correlation degrees of freedom. In other words, one should expect sizeable correlation effects not only from unfilled $3 \mathrm{~d}$ - or oxygen $2 \mathrm{p}$ shells, but from completely filled $\mathrm{O} 2 \mathrm{p}^{6}$ shell! In order to relevantly describe such a non-rigid atomic background and its coupling to the valent hole one might use a concept of the well-known "shell-droplet" model for nuclei after Bohr and Mottelson. ${ }^{22}$ In accordance with the model a set of completely filled electron shells which form an atomic background or vacuum state for a hole representation is described by certain internal collective degrees of freedom and a number of physical quantities such as electric quadrupole and magnetic moments. Valent hole(s) moves around this non-rigid background with strong interaction inbetween. Such an approach strongly differs from the textbook one that implies a rigid atomic orbital basis irrespective of varying filling number and external potential. 
None of the effective many-body Hamiltonians that are most widely used to study the effect of electron correlation in solids such as the Hubbard model, the Anderson impurity and lattice models, the Kondo model, contain this very basic and fundamental aspect of electron correlation that follows from the atomic analysis ${ }^{23}$ The Hubbard on-site repulsion U between opposite spin electrons on the same atomic orbital is widely regarded to be the only important source of electron correlation in solids. It is a clear oversimplification, and we need in a more realistic atomic models to describe these effects, especially for atoms in a specific external potential giving rise to a Coulomb instability. To this end we have proposed a generalized non-rigid shell model (see Refs 724 25). The model represents a variational method for the many-electron atomic configurations with the trial parameters being the coordinates of the center of the oneparticle atomic orbital. The resulting displacement of the atomic orbitals allows a simple interpretation of the electron density redistribution stemmed from taking into account the electron-electron repulsion, and the symmetry of a system can be readily used for the construction of the trial many-electron wave function. As a whole, the model bears a strong resemblance to the conventional well-known shell model by Dick and Overhauser ${ }^{26}$ widely used in lattice dynamics. In frames of the model the ionic configuration with filled electron shells is considered to be constituted of an outer spherical shell of $2(2 \mathrm{l}+1)$ electrons and a core consisting of the nucleus and the remaining electrons. In an electric field the rigid shell retains its spherical charge distribution but moves bodily with respect to the core. The polarizability is made finite by a harmonic restoring force of spring constant $k$ which acts between the core and shell. The shells of two ions repel one another and tend to become displaced with respect to the ion cores because of this repulsion. The respective displacement vector appears to be a simplest collective coordinate which specifies the change of the electron-nucleus attraction. It should be noted that such a displacement does not imply any variation in electronelectron repulsion and respective correlation energy.

However, as we shall see below, a simple shell model can be easily generalized to take account of correlation effects. To this end we must consider the displacements of separate one-electron orbitals to form the set of the variational parameters in a correlation function. Then we can introduce both the displacement of the center of "gravity" for filled shell and a set of the relative displacements of separate one-electron orbitals with regard to each other. The former form an "acoustical" mode and are described in frames of conventional shell model, while the latter form different novel "optical" modes. Such a seemingly naive non-rigid shell picture can provide both the microscopic substantiation of the conventional shell model and its generalization. Moreover, this non-rigid shell model points to a physically clear procedure to account for the correlation effects. Indeed, the "optical" displacement mode is believed to provide the minimal electron-electron repulsion. The non-rigid shell represents a novel specific atomic state that can be remarkably realized near a Coulomb instability.

The idea of displaced atomic shells has appeared many years $\operatorname{ago}^{27}$ in the very beginning of the quantum chemical era, and reflected the naive picture of the repelling electrons. However, the physically sound idea did not receive the relevant position in the hierarchy of correlation effects.

\section{Electron-lattice relaxation effects}

As it is mentioned above, the minimal energy cost of the optically excited disproportionation or electron-hole formation in insulating cuprates is $2.0-2.5 \mathrm{eV}$. However, the question arises, what is the energy cost for the thermal excitation of such a local disproportionation? The answer implies first of all the knowledge of relaxation energy, or the energy gain due to the lattice polarization by the localized charges. The full polarization energy $R$ includes the cumulative effect of electronic and ionic terms, associated with the displacement of electron shells and ionic cores, respectively ${ }^{4}$ The former term $R_{o p t}$ is due to the non-retarded effect of the electronic polarization by the momentarily localized electron-hole pair given the ionic cores fixed at their perfect crystal positions. Such a situation is typical for lattice response accompanying the Franck-Condon transitions (optical excitation, photoionization). On the other hand, all the long-lived excitations, i.e., all the intrinsic thermally activated states and the extrinsic particles produced as a result of doping, injection or optical pumping should be regarded as stationary states of a system with a deformed lattice structure. These relaxed states should be determined from the condition that the system energy has a local minimum when account is taken of the interaction of the electrons and holes with the lattice deformations. At least, it means that we cannot, strictly speaking, make use of the same energy parameters to describe the optical (e.g. photoexcited) hole and thermal (e.g. doped) hole.

For the illustration of polarization effects in cuprates we apply the shell model calculations to look specifically at energies associated with the localized holes of $\mathrm{Cu}^{3+}$ and $\mathrm{O}^{-}$in "parent" $\mathrm{La}_{2} \mathrm{CuO}_{4}$ compound. It follows from these calculations that there is a large difference in the lattice relaxation energies for $\mathrm{O}^{-}$and $\mathrm{Cu}^{3+}$ holes. The lattice relaxation energy, $-\Delta R_{t h}^{\alpha}$, caused by the hole localization at the $\mathrm{O}$-site $(4.44 \mathrm{eV})$ appears to be significantly larger than that for the hole localized at the $\mathrm{Cu}$-site $(2.20$ $\mathrm{eV})$. This indicates the strong electron-lattice interaction in the case of the hole localized at the O-site and could suggest that the hole trapping is more preferential in the oxygen sublattice. In both cases we deal with the several eV-effect both for electronic and ionic contributions to relaxation energy. Moreover, such an estimation seems to be typical for different insulators $\stackrel{4,5}{*}$ It is worth noting that the electron-lattice interaction is believed to be 
one of the main sources of correlated particle hopping resulting in different probabilities for two types of a charge transfer.

\section{Generalized Peierls-Hubbard model and "negative-U" effect}

Transition metal oxides with strong electron and lattice polarization effects need in a revisit of many conventional theoretical concepts and approaches. In particular, we should modify conventional Hubbard model as it is done, for instance, in a "dynamic" Hubbard model by $\mathrm{Hirsch}^{23}$ or a modified Peierls-Hubbard model 18 with a classical description of the anharmonic core/shell displacements. Having in mind the application to insulating cuprates let address a square lattice Hubbard model with a half-filling and a strong on-site coupling of valent hole with core/shell displacements, which is described by the following Hamiltonian

$$
\begin{aligned}
\hat{H} & =-\sum_{<i, j>, \sigma} t(i j) \hat{c}_{i \sigma}^{\dagger} \hat{c}_{j \sigma}+U \sum_{i, \sigma \sigma^{\prime}} n_{i \sigma} n_{i \sigma^{\prime}} \\
& +\sum_{i} v_{a n}\left(q_{i}, n_{i}\right)+\sum_{<i, j>} v_{i n t}\left(q_{i}, q_{j}\right),
\end{aligned}
$$

where $\hat{c}_{i \sigma}^{\dagger}\left(\hat{c}_{j \sigma}\right)$ are creation (annihilation) operators for valent hole; $t(i j)=t\left(q_{i}, q_{j}\right)$ is the transfer integral between two neighboring lattice sites which depends on the dimensionless core/shell displacement coordinate; $U$ is the on-site repulsion energy; $v_{a n}\left(q_{i}, n_{i}\right)$ is the configurational energy that incorporates the coupling between valent holes and the site-localized anharmonic core/shell mode with dimensionless displacement coordinate $q_{i}$;

$$
v_{a n}\left(q_{i}, n_{i}\right)=a\left(n_{i}\right) q_{i}^{2}-b\left(n_{i}\right) q_{i}^{4}+c\left(n_{i}\right) q_{i}^{6},
$$

where $a, b, c$ are the functions of the hole occupation number such as

$$
a\left(n_{i}\right)=a_{0}+a_{1} n_{i}+a_{2} n_{i}^{2},
$$

It is clear that $v_{a n}\left(q_{i}, n_{i}\right)$ includes the renormalization both of the one-particle energy and the on-site hole-hole repulsion. The last term in (22) represents the intersite configurational coupling. The $q$-dependence of transfer integral implies the correlated character of the hole hopping, and can be transformed into the effective dependence on hole occupation number ${ }^{23}$

$$
t\left(n_{i}, n_{j}\right)=t\left(1+\alpha\left(n_{i}+n_{j}\right)+\beta n_{i} n_{j}\right) .
$$

with $\alpha, \beta$ being the correlated hopping parameters.

The conventional Hubbard Hamiltonian, or $t$ - $U$-model, stabilizes the spin density wave (SDW) electron order with $n_{i}=1$. In a strongly correlated limit $U \geq t$ the
Hubbard model reduces to a Heisenberg antiferromagnetic model. Depending on the parameters of the holeconfigurational coupling and correlated hopping the modified Hubbard Hamiltonian (22) can stabilize the "disproportionated" or charge ordered $(\mathrm{CO})$ electron phase with the on-site filling numbers $n=0$, and $n=2$ thus leading to the "negative-U" effect. Even simple modified model turns out to be very complicated and leads to a very rich physics ${ }^{23}$ Depending on the values of parameters the system yields the SDW phase with no core/shell displacements as a true ground state with a global minimum of free energy, and CO phase with shell displacements as a false ground state with a local minimum, or vice versa $\stackrel{\underline{8}}{=}$ Strong anharmonicity $v_{a n}\left(q_{i}, n_{i}\right)$ makes possible phase transitions between the phases the first order ones.

\section{Vibronic reduction of charge transfer integrals}

In general, charge, spin and vibronic modes are strongly coupled and so we have to do with the hybrid modes. For a weak intermode coupling regime the charge transfer is accompanied by the induced local structural fluctuations, that provides the vibronic reduction of the charge transfer integral:

$$
t_{12}=t_{12}^{(0)} K_{v i b} \quad K_{v i b}=\left\langle\chi_{1} \mid \chi_{2}\right\rangle^{2},
$$

where $K_{v i b}$ is a vibronic reduction factor, $\left\langle\chi_{1} \mid \chi_{2}\right\rangle$ is an overlap integral for the local oscillatory states with and without particle transferred. In an opposite regime of the strong intermode coupling one assumes that different electronic parameters for the $e$ - and $h$-centers are distinguished significantly up to different type of the adiabatic potential and appropriate JT mode. This regime favors the charge localization.

The vibronic reduction factor $K_{v i b}$, or the FranckCondon factor ${ }^{28}$ may be written as follows

$$
K_{v i b}=N \exp (-\gamma)
$$

where $N$ and $\gamma$ in a complicated manner depend on the vibronic constants, the oxygen and 3d-metal atomic masses. For a simplest one-dimensional single-mode case

$$
K_{v i b}=\frac{2 \tau}{1+\tau^{2}} \exp \left(-\frac{(\Delta Q)^{2}}{l_{1}^{2}+l_{2}^{2}}\right),
$$

where $l_{1}$ and $l_{2}$ are the effective oscillatory lengths of the 1- and 2-centers, respectively, $\tau=l_{1} / l_{2}, \Delta Q$ is the distance separating the minima of the adiabatic potential for the 1- and 2-centers.

\section{Spin reduction of charge transfer integrals}

Overall the paper we neglect a spin degree of freedom which can crucially impact on the charge transport. The 
most part of 3d oxides are characterized by an antiferromagnetic spin background that implies a localization effect due to strong spin reduction of one-particle transfer integrals and the probability amplitude for a polar center transfer $M^{ \pm}+M^{0} \rightarrow M^{0}+M^{ \pm}$or the motion of the electron (hole) center in the matrix of $M^{0}$-centers. From the other hand, in antiferromagnets there is no problems with another type of the CT which specifies the probability amplitude for a local disproportionation, or the spinsinglet $e h$-pair creation: $M^{0}+M^{0} \rightarrow M^{ \pm}+M^{\mp}$, and the inverse process of the spin-singlet $e h$-pair recombination: $M^{ \pm}+M^{\mp} \rightarrow M^{0}+M^{0}$. In other words, the spin subsystem can strongly affect the correlated character of the charge transfer leading to unconventional situations like that of spin-singlet eh-pairs moving through the lattice freely without disturbing the antiferromagnetic spin background, in contrast to the single particle motion. So, it seems that the situation in antiferromagnetic $3 \mathrm{~d}$ insulators may differ substantially from that in usual semiconductors or in other bandlike insulators where, as a rule, the effective mass of the electron-hole pair is larger than that of an unbound electron and hole.

\section{S=1 PSEUDOSPIN FORMALISM FOR MODEL MIXED VALENCE SYSTEM}

\section{A. Pseudospin operators}

The problem of the multi-stability of solids looks rather trivial when one say about the orbital and/or spin degrees of freedom. Usually in such a case we start from the lattice of coupled orbital and/or spin momenta described by the relevant (spin-)Hamiltonian that implies the variety of possible collective orbital and/or spin orderings that compete with each other under different external conditions. In other words, the multi-stability accompanies the basic degeneracy inherent to a certain atom, ion, or center with a nonzero orbital and/or spin momentum. Such an outlook is believed to be easily extended to systems with charge degree of freedom which can be represented to be a system of either centers which possible charge states form a pseudo-multiplet. Below we address a simple model of a mixed-valence system with three possible stable valent states of a cation-anionic cluster, hereafter $M: M^{0}, M^{ \pm}$, forming the charge (isospin) triplet. Starting from $M^{0}$ state as a bare vacuum state, we may address the $M^{ \pm}$centers as a result of pseudospin $\Delta S_{z}= \pm 1$ deviation, or as a hole and electron, respectively. Below we intend to concentrate themselves on charge degree of freedom, and that is why we neglect the orbital, spin, and lattice degrees of freedom. It implies a renormalization of different parameters, mainly it concerns the charge transfer.

Similarly to the neutral-to-ionic electronic-structural transformation in organic charge-transfer crystals (see paper by T. Luty in Ref 11) the system of charge triplets can be described in frames of the $\mathrm{S}=1$ pseudo-spin formalism. To this end we associate three charge states of the $M$-center with different valence: $M^{0}, M^{ \pm}$with three components of $S=1$ pseudo-spin (isospin) triplet with $M_{S}=0,+1,-1$, respectively.

The $S=1$ spin algebra includes three independent irreducible tensors $\hat{V}_{q}^{k}$ of rank $k=0,1,2$ with one, three, and five components, respectively, obeying the WignerEckart theorem 29

$$
\left\langle S M_{S}\left|\hat{V}_{q}^{k}\right| S M_{S}^{\prime}\right\rangle=(-1)^{S-M_{S}}\left(\begin{array}{ccc}
S & k & S \\
-M_{S} & q & M_{S}^{\prime}
\end{array}\right)\left\langle S\left\|\hat{V}^{k}\right\| S\right\rangle .
$$

Here we make use of standard symbols for the Wigner coefficients and reduced matrix elements. In a more conventional Cartesian scheme a complete set of the non-trivial pseudo-spin operators would include both $\mathbf{S}$ and a number of symmetrized bilinear forms $\left\{S_{i} S_{j}\right\}=$ $\left(S_{i} S_{j}+S_{j} S_{i}\right)$, or spin-quadrupole operators, which are linearly coupled to $V_{q}^{1}$ and $V_{q}^{2}$, respectively

$$
\begin{gathered}
V_{q}^{1}=S_{q} ; S_{0}=S_{z}, S_{ \pm}=\mp \frac{1}{\sqrt{2}}\left(S_{x} \pm i S_{y}\right): \\
V_{0}^{2} \propto\left(3 S_{z}^{2}-\mathbf{S}^{2}\right), V_{ \pm 1}^{2} \propto\left(S_{z} S_{ \pm}+S_{ \pm} S_{z}\right), V_{ \pm 2}^{2} \propto S_{ \pm}^{2} .
\end{gathered}
$$

These pseudo-spin operators are not to be confused with real physical spin-operators; they act in an imaginary pseudo-space.
To describe different types of pseudo-spin ordering in a mixed-valence system we have to introduce eight order parameters: two diagonal order parameters $\left\langle S_{z}\right\rangle$ and $\left\langle S_{z}^{2}\right\rangle$, and six off-diagonal order parameters $\left\langle V_{q}^{k}\right\rangle$ $(q \neq 0)$. Two former order parameters can be termed as valence and ionicity, respectively. The off-diagonal order parameters describe different types of the valence mixing. Indeed, operators $V_{q}^{k}(q \neq 0)$ change the $z$ projection of pseudo-spin and transform the $\left|S M_{S}\right\rangle$ state into $\left|S M_{S}+q\right\rangle$ one. In other words, these can change valence and ionicity. It should be noted that for the $S=1$ pseudospin algebra there are two operators: $V_{+1}^{1}$ and $V_{ \pm 1}^{2}$, that change the pseudo-spin projection by \pm 1 , with slightly different properties

$$
\left\langle 0\left|\hat{S}_{ \pm}\right| \mp 1\right\rangle=\left\langle \pm 1\left|\hat{S}_{ \pm}\right| 0\right\rangle=\mp 1,
$$


but

$$
\left\langle 0\left|\left(S_{z} S_{ \pm}+S_{ \pm} S_{z}\right)\right| \mp 1\right\rangle=-\left\langle \pm 1\left|\left(S_{z} S_{ \pm}+S_{ \pm} S_{z}\right)\right| 0\right\rangle=+1 .
$$

\section{B. Gell-Mann operators and generalized pseudospin Hamiltonian}

Three spin-linear (dipole) operators $\hat{S}_{1,2,3}$ and five independent spin-quadrupole operators $\left\{\hat{S}_{i}, \hat{S}_{j}\right\}-\frac{2}{3} \hat{\mathbf{S}}^{2} \delta_{i j}$ given $S=1$ form eight Gell-Mann operators being the generators of the $\mathrm{SU}(3)$ group. Below we will make use of the appropriate Gell-Mann $3 \times 3$ matrices $\Lambda^{(k)}$, which differ from the conventional $\lambda^{(k)}$ only by a renumeration ${ }^{30}$ $\lambda^{(1)}=\Lambda^{(6)}, \lambda^{(2)}=\Lambda^{(3)}, \lambda^{(3)}=\Lambda^{(8)}, \lambda^{(4)}=\Lambda^{(5)}$, $\lambda^{(5)}=-\Lambda^{(2)}, \lambda^{(6)}=\Lambda^{(4)}, \lambda^{(7)}=\Lambda^{(1)}, \lambda^{(8)}=\Lambda^{(7)}$. First three matrices $\Lambda^{(1,2,3)}$ correspond to linear (dipole) spin operators:

$$
\Lambda^{(1)}=S_{x} ; \quad \Lambda^{(2)}=S_{y} ; \quad \Lambda^{(3)}=S_{z}
$$

while other five matrices correspond to quadratic (quadrupole) spin operators:

$$
\begin{gathered}
\Lambda^{(4)}=-\left\{S_{z} S_{y}\right\} ; \quad \Lambda^{(5)}=-\left\{S_{x} S_{z}\right\} ; \quad \Lambda^{(6)}=-\left\{S_{x} S_{y}\right\} ; \\
\Lambda^{(7)}=-\frac{1}{\sqrt{3}}\left(S_{x}^{2}+S_{y}^{2}-2 S_{z}^{2}\right) ; \quad \Lambda^{(8)}=S_{y}^{2}-S_{x}^{2} ; \\
S_{x}^{2}+S_{y}^{2}+S_{z}^{2}=2 \hat{E}
\end{gathered}
$$

with $\hat{E}$ being a unit $3 \times 3$ matrix.

The generalized spin- 1 model can be described by the Hamiltonian bilinear on the $\mathrm{SU}(3)$-generators $\Lambda^{(k)}$

$$
\hat{H}=-\sum_{i, \eta} \sum_{k, m=1}^{8} J_{k m} \hat{\Lambda}_{i}^{(k)} \hat{\Lambda}_{i+\eta}^{(m)} .
$$

Here $i, \eta$ denote lattice sites and nearest neighbors, respectively. This is a $S=1$ counterpart of the $S=1 / 2$ model Heisenberg Hamiltonian with three generators of the $\mathrm{SU}(2)$ group or Pauli matrices included instead of eight Gell-Mann matrices.

\section{Generalized mean-field model}

In frames of a classical, or mean-field description of the $S=1$ quantum pseudo-spin system we start from a coherent state approximation with trial functions ${ }^{30}$

$$
\psi=\prod_{j \in \text { lattice }} c_{i}(j) \psi_{i}=\prod_{j \in \text { lattice }}\left(a_{i}(j)+i b_{i}(j)\right) \psi_{i} .
$$

Here $j$ labels a lattice site and the spin functions $\psi_{i}$ in a Cartesian basis are used: $\psi_{z}=\mid 10>$ and $\psi_{x, y} \sim(\mid 11>$ $\pm \mid 1-1>) / \sqrt{2}$. The linear (dipole) pseudo-spin operator within $|x, y, z\rangle$ basis is represented by a simple matrix:

$$
<\psi_{i}\left|S_{j}\right| \psi_{k}>=-i \varepsilon_{i j k},
$$

and for the order parameters one easily obtains:

$$
<\hat{\mathbf{S}}>=-2[\mathbf{a} \times \mathbf{b}] ;<\left\{\hat{S}_{i} \hat{S}_{j}\right\}>=2\left(\delta_{i j}-a_{i} a_{j}-b_{i} b_{j}\right)
$$

given the normalization constraint $\mathbf{a}^{2}+\mathbf{b}^{2}=1$. Thus, for the case of spin- 1 system the order parameters are determined by two classical vectors (two real components of one complex vector $\mathbf{c}=\mathbf{a}+\mathbf{i b}$ from (20) ). The two vectors are coupled, so the minimal number of dynamic variables describing the $S=1$ spin system appears to be equal to four. Along with $\mathbf{a}, \mathbf{b}$ vectors one might introduce $\mathbf{l}=[\mathbf{a} \times \mathbf{b}]$. Hereafter we would like to emphasize the director nature of the $\mathbf{c}$ vector field: $\psi(\mathbf{c})$ and $\psi(-\mathbf{c})$ describe the physically identical states. It is worth noting that the coherent states provide the optimal way both to a correct mean-field approximation (MFA) and respective continuous models $\underline{30}$

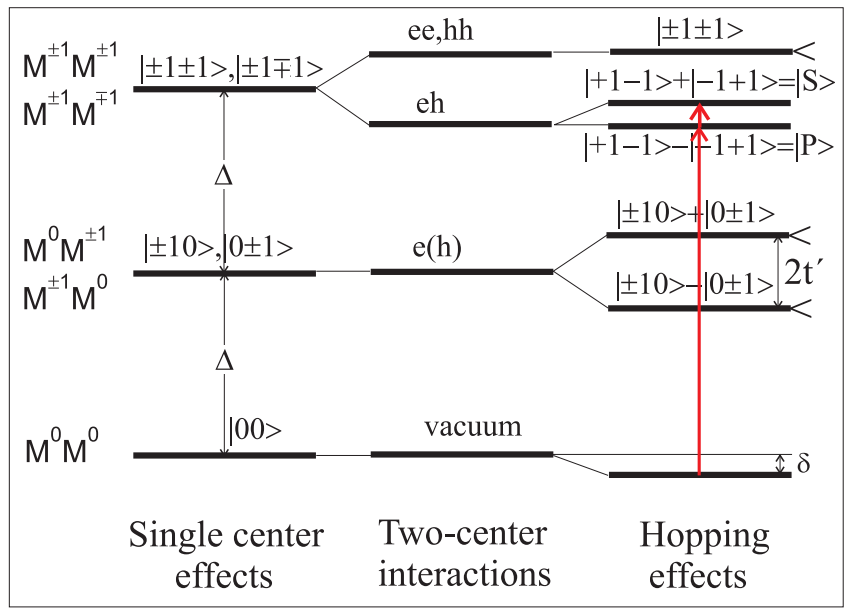

FIG. 2: Schematic energy spectrum of two $n n$ M-centers system (see text for details). Arrows mark the dipole-allowed CT transitions.

\section{MODEL MIXED-VALENCE SYSTEM}

\section{A. Effective pseudo-spin Hamiltonian}

Effective pseudo-spin Hamiltonian for our model mixed-valence system should incorporate a large number of contributions that describe different long- and short-range coupling between $M^{0, \pm}$ centers, single-ion and two-ion terms. Single-site terms can be subdivided into single-ion anisotropy and pseudo-Zeeman interaction. Bilinear and biquadratic two-site terms can be subdivided into diagonal interactions like "density-density", and off-diagonal terms that describe charge fluctuations 
conserving the total charge of the system, such as oneelectron(hole) and two-electron(hole) transport. An effective pseudo-spin Hamiltonian of the model mixedvalence system which takes into account the main part of aforementioned contributions can be represented as follows

$$
\hat{H}=\sum_{i}\left(\Delta_{i} S_{i z}^{2}-h_{i} S_{i z}\right)+\sum_{<i, j>} v_{i j} S_{i z}^{2} S_{j z}^{2}+\sum_{<i, j>} V_{i j} S_{i z} S_{j z}+
$$$$
\sum_{<i, j>}\left[D_{i j}^{(1)}\left(S_{i+} S_{j-}+S_{i-} S_{j+}\right)+D_{i j}^{(2)}\left(T_{i+} T_{j-}+T_{i-} T_{j+}\right)\right]
$$$$
+\sum_{<i, j>} t_{i j}\left(S_{i+}^{2} S_{j-}^{2}+S_{i-}^{2} S_{j+}^{2}\right)
$$

where

$$
T_{ \pm}=\left(S_{z} S_{ \pm}+S_{ \pm} S_{z}\right) .
$$

Two first single-ion terms describe the effects of bare pseudo-spin splitting, or the local energy of $M^{0, \pm}$ centers. Interestingly, the parameter $\Delta$ can be related with correlation Hubbard parameter $U: U=2 \Delta$. The second term may be associated with an external, generally speaking, non-uniform pseudo-magnetic field $h_{i}$, in particular, a real electric field. It is easy to see that it describes an electron/hole assymetry. The third and fourth terms describe the effects of long- and short-range interionic interaction including screened Coulomb and covalent coupling.

If to apply the familiar spin terminology, the first term in (22) represents a single-ion anisotropy, the second does the Zeeman term, the fourth and fifth do the anisotropic Heisenberg exchange, and the third and sixth do the biquadratic spin-quadrupolar coupling. To illustrate the role of different terms in (22) we present in Fig.1 a schematic energy spectrum of $n n$ pair of $M$ centers provided an eh-symmetry $(h=0)$ and $|00\rangle$ ground state $(\Delta>0)$. It is worth noting the effect of a renormalization of the ground state due to eh-pair creation/recombination effect $\left(t_{i j}^{\prime \prime} \neq 0\right)$ with a stabilization energy $\delta \approx\left|t_{i j}^{\prime \prime}\right|^{2} / 2 \Delta$. Two electron-hole states with S(even) and P- (odd) type symmetry have a very strong dipole coupling with the large value of $S-P$ transition dipole matrix element:

$$
d=|\langle S|\hat{\mathbf{d}}| P\rangle| \approx 2 e R_{M M} \approx 2 e \times 4 \AA .
$$

Contrary to $P$-type pair state the $S$-type one is dipoleforbidden and corresponds to a so-called two-photon state.

One should note that despite many simplifications, and first the neglect of orbital and spin degrees of freedom, quenched lattice approximation, the effective Hamiltonian (22) is rather complex, and represents one of the most general forms of the anisotropic $S=1$ nonHeisenberg Hamiltonians. For the system there are two classical (diagonal) order parameters: $\left\langle S_{z}\right\rangle=n$ being a valence, or charge density with electro-neutrality constraint $\sum_{i} n_{i}=\sum_{i} S_{i z}=0$, and $\left\langle S_{z}^{2}\right\rangle=n_{p}$ being the density of polar centers $M^{ \pm}$, or "ionicity". In addition, there are two unconventional off-diagonal order parameters: "fermionic" $\left\langle S_{+}\right\rangle$and "bosonic " $\left\langle S_{+}^{2}\right\rangle$; the former describes a phase ordering for the disproportionation reaction, or the single-particle transfer, while the latter does for exchange reaction, or for the two-particle transfer. Indeed, the $\hat{S}_{+}$operator creates a hole and is fermionic in nature, whereas the $\hat{S}_{+}^{2}$ does a hole pair, and is bosonic in nature.

\section{B. Single and two-particle transport}

The last three terms in (22) representing the one- and two-particle hopping, respectively, are of primary importance for the transport properties, and deserve special interest.

Two types of one-particle hopping are governed by two transfer integrals $D^{(1,2)}$, respectively. In accordance with (17) and (18) the transfer integral $t_{i j}^{\prime}=\left(D_{i j}^{(1)}+D_{i j}^{(2)}\right)$ specifies the probability amplitude for a local disproportionation, or the eh-pair creation

$$
M^{0}+M^{0} \rightarrow M^{ \pm}+M^{\mp}
$$

and the inverse process of the eh-pair recombination

$$
M^{ \pm}+M^{\mp} \rightarrow M^{0}+M^{0},
$$

while the transfer integral $t_{i j}^{\prime \prime}=\left(D_{i j}^{(1)}-D_{i j}^{(2)}\right)$ specifies the probability amplitude for a polar center transfer

$$
M^{ \pm}+M^{0} \rightarrow M^{0}+M^{ \pm},
$$

or the motion of the electron (hole) center in the matrix of $M^{0}$-centers or motion of the $M^{0}$-center in the matrix of $M^{ \pm}$-centers. It should be noted that, if $t_{i j}^{\prime \prime}=0$ but $t_{i j}^{\prime} \neq 0$, the eh-pair is locked in two-site configuration.

The two-electron(hole) hopping is governed by transfer integral $t_{i j}$, or a probability amplitude for the exchange reaction:

$$
M^{ \pm}+M^{\mp} \rightarrow M^{\mp}+M^{ \pm} .
$$

or the motion of the electron (hole) center in the matrix of hole (electron) centers.

It is worth noting that in Hubbard-like models all the types of one-electron(hole) transport are governed by the same transfer integral: $t_{i j}^{\prime}=t_{i j}^{\prime \prime}=t_{i j}$, while our model implies independent parameters for a disproportionation/recombination process and simple quasiparticle motion in the matrix of $M^{0}$-centers. In other words, we deal with a "correlated" single particle transport $\underline{\underline{6}}$ 


\section{Mean-field approximation:three generic MFA-phases}

First of all we would like to emphasize the difference between classical and quantum mixed-valence systems. Classical (or chemical) description implies the neglect of the off-diagonal purely quantum CT effects: $D^{(1,2)}=t=0$, hence the valence of any site remains to be definite: $0, \pm 1$, and we deal with a system of localized polar centers. In quantum systems with a nonzero charge transfer we arrive at quantum superpositions of different valence states resulting in indefinite on-site valence and ionicity which effective, or mean values $\left\langle S_{z}\right\rangle$ and $\left\langle S_{z}^{2}\right\rangle$ can vary from -1 to +1 and 0 to +1 , respectively.

Making projection of the effective pseudo-spin Hamiltonian for the system onto a space of states like (20), we obtain an energy functional which equivalent to a classical energy of the two coupled vector $(\mathbf{a}, \mathbf{b})$ fields defined on the common lattice. Thus, in the framework of the pseudo-spin $S=1$ centers model when the collective wave function is represented to be a product of the site functions like (20), the quantum problem is reduced to a classical variation problem for a minimum of the energy for two coupled vector fields.

In frames of mean-field approximation (MFA) we may make use of coherent states (20) that provide a physically clear assignment of different phases with a straightforward recipe of its qualitative and quantitative analysis. All the MFA-phases one may subdivide into those with a definite and indefinite ionicity, respectively. There are two MFA-phases with definite ionicity; 0 :

1) Insulating monovalent $M^{0}$-phase with $\left\langle S_{z}^{2}\right\rangle=$

The $M^{0}$-phase is specified by a simple uniform arrangement of $\mathbf{a}$ and $\mathbf{b}$ vectors parallel to $z$-axis: $\mathbf{a}\|\mathbf{b}\| O_{z}$. In such a case the on-site wave function is specified by unit vector (a, or $\mathbf{b})$ parallel to $z$-axis. It is a rather conventional ground state phase for various charge transfer insulators such as oxides with a positive magnitude of $\Delta$ parameter $(U>0)$. All the centers have the same bare $M^{0}$ valence state. In other words, the $M^{0}$ phase is characterized both by definite site ionicity and valence. So, all the order parameters turn into zero: $\left\langle S_{z}\right\rangle=\left\langle S_{z}^{2}\right\rangle=\left\langle S_{+}\right\rangle=\left\langle S_{+}^{2}\right\rangle=0$. This is an "easy-plane" phase for the pseudo-spins, but an "easy-axis" one for the $\mathbf{a}$ and/or $\mathbf{b}$ vectors.

2) Mixed-valence binary (disproportionated) $M^{ \pm}$-phase with $\left\langle S_{z}^{2}\right\rangle=1$ :

This phase usually implies an overall disproportionation $M^{0}+M^{0} \rightarrow M^{ \pm}+M^{\mp}$ that seems to be realizable if $\Delta$ parameter becomes negative one (negative $U<0$ effect). It is a rather unconventional phase for insulators. All the centers have the "ionized" valence state, one half the $M^{+}$state, and another half the $M^{-}$one, though one may in common conceive of deviation from fifty-fifty distribution. A simplified "chemical" approach to $M^{ \pm}$-phase as to a classical disproportionated phase is widely spread in solid state chemistry ${ }^{14}$ In contrast with the $M^{0}$ phase the $M^{ \pm}$-phase is specified by a planar orientation of $\mathbf{a}$ and $\mathbf{b}$ vectors $\left(\mathbf{a}, \mathbf{b} \perp O_{z}\right)$ with $\mathbf{a}$ varied angle in between. There is no fermionic transport: $\left\langle S_{+}\right\rangle=0$, while the bosonic one may exist, and, in common, $\left\langle S_{+}^{2}\right\rangle=-\cos \left(\phi_{a}-\phi_{b}\right) e^{i\left(\phi_{a}+\phi_{b}\right)} \neq 0$. This is an "easy-axis" phase for the pseudo-spins, but an "easyplane" one for the $\mathbf{a}$ and $\mathbf{b}$ vectors.

The mixed valence $M^{ \pm}$phase as a system of strongly correlated electron and hole centers is equivalent to the lattice hard-core Bose system with an inter-site repulsion, or electron-hole Bose liquid (EHBL) in contrast with $\mathrm{EH}$ liquid in conventional semiconductors like Ge, Si where we deal with a two-component Fermi-liquid. Indeed, one may address the electron $M^{-}$center to be a system of a local boson $\left(e^{2}\right)$ localized on the hole $M^{+}$center: $M^{-}=$ $M^{+}+e^{2}$.

In accordance with this analogy we assign three well known molecular-field uniform phase states of the $M^{ \pm}$ binary mixture:

i) charge ordered (CO) insulating state with $\left\langle S_{z}\right\rangle= \pm 1, \mathbf{a} \perp \mathbf{b}$, and zero modulus of bosonic offdiagonal order parameter: $\left|\left\langle S_{+}^{2}\right\rangle\right|=0$;

ii) Bose-superfluid (BS) superconducting state with $\left\langle S_{z}\right\rangle=0$, a and $\mathbf{b}$ being collinear, $\left\langle S_{+}^{2}\right\rangle=e^{2 i \phi}$;

iii) mixed Bose-superfluid-charge ordering (BS+CO) superconducting state (supersolid) with $0<\left|\left\langle S_{z}\right\rangle\right|<1$, a and $\mathbf{b}$ being oriented in $x y$-plane, but not collinear, $\left\langle S_{+}^{2}\right\rangle=-\cos \left(\phi_{a}-\phi_{b}\right) e^{i\left(\phi_{a}+\phi_{b}\right)} \neq 0$.

In addition, we should mention the high-temperature non-ordered (NO) Bose-metallic phase with $\ll S_{z} \gg=0$.

Rich phase diagram of $M^{ \pm}$binary mixture with unconventional superfluid and supersolid regions looks tempting, however, actually, their stabilization requires strong suppression of Coulomb repulsion between electron (hole) centers. Despite significant screening effect, the stabilization of uniform $\mathrm{BS}$ or $\mathrm{BS}+\mathrm{CO}$ superconducting state as a result of a disproportionation reaction in a bare insulator $^{14}$ seems to be unrealistic.

3) Mixed-valence ternary ("underdisproportionated") $M^{0, \pm}$-phase:

For two preceding cases the order parameter $\left\langle S_{z}^{2}\right\rangle$, or ionicity relates to its limiting values (0 or 1 , respectively). For the MFA-phase with indefinite ionicity, or mixed-valence ternary ("under-disproportionated") $M^{0, \pm}$-phase, $0<\left\langle S_{z}^{2}\right\rangle<1$, that is we have a mixture of the $M^{0}, M^{ \pm}$centers. From the viewpoint of the classical $\mathbf{a}, \mathbf{b}$ vectors formalism the phase corresponds to the arbitrarily space-oriented $\mathbf{l}=[\mathbf{a} \times \mathbf{b}]$ vector. Both offdiagonal order parameters, fermionic $\left\langle S_{+}\right\rangle$and bosonic $\left\langle S_{+}^{2}\right\rangle$ are, in common, non-zero, albeit with some correlation in between. So, for the ternary system one expects a coexistence of fermionic and bosonic transport.

It should be noted that a complete pseudo-spin description of the two last model mixed-valence systems implies a two-sublattice approximation to be a minimal model compatible with a sign of Coulomb interaction and a respective tendency towards the checkerboard-like charge ordering. 


\section{INSULATING MONOVALENT $M^{0}$-PHASE}

Insulating monovalent $M^{0}$-phase is a typical one for the ground state of insulating transition metal oxides, or Mott-Hubbard insulators. It is worth noting that in frame of conventional band model approach the $M^{0}$ phase, e.g., in parent cuprates, is associated with a metallic half-filled hole band system. Below we address different types of quasiparticle excitations in such a system focusing on the features of the correlated hopping, governed generally by two transfer integrals $t_{i j}^{\prime}$ and $t_{i j}^{\prime \prime}$ which competition results in unconventional properties of electrons and holes in bare insulating monovalent $M^{0}$-phase. We show that $M^{0}$-phase can be unstable with regard to the self-trapping of CT excitons and nucleation of droplets of EH Bose liquid.

\section{A. Electron, hole, and electron-hole excitations}

Starting from monovalent $M^{0}$-phase as a vacuum state $|0\rangle$ we introduce an electron-hole representation where $M_{i}^{-}$center is derived as a result of an electron creation $\hat{a}_{i}^{\dagger}|0\rangle$, and $M_{i}^{+}$center is derived as a result of a hole creation $\hat{b}_{i}^{\dagger}|0\rangle$. Then we transform pseudo-spin Hamiltonian (22) into that of a system of effective electrons and holes

$$
\begin{gathered}
\hat{H}=\Delta \sum_{i}\left(n_{i}^{h}+n_{i}^{e}\right)+\sum_{\langle i j\rangle} t_{i j}\left(\hat{a}_{i}^{\dagger} \hat{a}_{j}+\hat{b}_{i}^{\dagger} \hat{b}_{j}\right)+ \\
\sum_{i, j}\left[V^{h h}(i j)\left(n_{i}^{h} n_{j}^{h}+V^{e e}(i j) n_{i}^{e} n_{j}^{e}+V^{e h}(i j)\left(n_{i}^{h} n_{j}^{e}+n_{j}^{h} n_{i}^{e}\right)\right]\right. \\
+\sum_{\langle i j\rangle} t_{i j}^{\prime \prime}\left(\hat{a}_{i}^{\dagger} \hat{b}_{j}^{\dagger}+\hat{a}_{i} \hat{b}_{j}\right)
\end{gathered}
$$

where $V_{i i} \rightarrow \infty$ to prohibit two-particle occupation of a single site. Here we suppose $h=0$ that provides an electron-hole symmetry. The first line in (24) represents the single particle (electrons/holes) terms, the second one does the interparticle coupling, while the third one describes the creation and annihilation (recombination) of eh-pairs. It is worth noting that the latter terms describe some sort of eh-coupling.

In terms of a pseudo-spin analogy the electrons and holes are associated with pseudo-spin $\Delta S_{z}= \pm 1$ deviations for an easy-plane magnet, localized or delocalized (pseudo-spin wave).

The behavior of electron/hole system crucially depends on the relation between two transfer integrals $t_{i j}^{\prime}, t_{i j}^{\prime \prime}$ Below we address two distinct limiting situations:

I. $t_{i j}^{\prime \prime}=0$ : Forbidden recombination/creation regime. In this case we deal with the bands of well defined electrons and holes with a charge gap $E_{g}^{e, h}=\Delta-z\left|t_{n n}^{\prime}\right|$ for both types of carriers. Optical gap for unbound eh-pairs is $E_{g}^{e h}=2 E_{g}^{e, h}$. However, in such a case we may expect for the formation of Wannier excitons, or eh-pairs bound due to a screened Coulomb eh-coupling. In terms of pseudo-spin formalism the Wannier excitons may be regarded as two pseudo-spin waves having formed a quasilocalized state due to a long-range antiferromagnetic Ising exchange: $V_{i j} S_{i z} S_{j z}$.

II. $t_{i j}^{\prime}=0$ : Regime of localized electrons and holes with a dimerization effect and well defined $n n$ eh-pairs, or CT excitons. In such a case the charge gap is $E_{g}^{e, h}=\Delta$ for both types of localized quasiparticles. As it is clearly seen in Fig. 1 the $t_{i j}^{\prime \prime} \neq 0$ hopping results in a dimerization effect with a quantum renormalization of the vacuum state and indefinite ionicity, the formation of two types of localized eh-pairs, or CT excitons of S- and P-type. In frames of our $n n$ approximation the CT excitons are localized. Optical gap is determined by the energy of P-type CT exciton: $E_{g}^{e h}=2 \Delta+\delta$.

Thus we arrive at two limiting types of monovalent $M^{0}$ insulators with a dramatic difference in behavior of electrons and holes, as well as electron-hole pairs. In type I insulators $\left(t_{i j}^{\prime} \gg t_{i j}^{\prime \prime}\right)$ we deal with well defined bands of electrons and holes forming Wannier excitons, while in type II insulators $\left(t_{i j}^{\prime \prime} \gg t_{i j}^{\prime}\right)$ we deal with localized electrons and holes which can form $n n$ eh-pairs, or CT excitons.

The most part of $3 \mathrm{~d}$ oxides are characterized by an antiferromagnetic spin background that implies strong spin reduction of one-particle transfer integrals $t_{i j}^{\prime}$. In other words, these, seemingly, belong to type II insulators, where spin-singlet CT excitons can move through the lattice freely without disturbing the antiferromagnetic spin background, in contrast to the single hole motion. So, it seems that the situation in antiferromagnetic $3 \mathrm{~d}$ insulators differs substantially from that in usual semiconductors or in other bandlike insulators where, as a rule, the effective mass of the electron-hole pair is larger than that of an unbound electron and hole.

The Wannier excitons are formed due to an ehcoupling, while the CT excitons are formed due to a $k i$ netic cutoff, or a specific feature of correlated hopping, in other words, the former have a potential, while the latter a kinetic nature.

It is worth noting that both M-centers within P-type CT excitons have a certain ionicity in contrast to S-type CT excitons which can mix with bare $M^{0} M^{0}$ ground state. CT excitons form peculiar quanta of a disproportionation reaction and may be viewed to be a minimal droplet of electron-hole Bose liquid.

In general, eh-excitations in $M^{0}$ phase consist of superpositions of pairs of free electrons and holes, and CT excitons. One expects two types of superpositions: CT exciton-like and band-like. The former have a localized character, while the latter have an itinerant one.

The nature of the optical excitations accompanied by creation of electron-hole pairs in $3 \mathrm{~d}$ oxides is not fully understood. One of the central issues in the analysis of electron-hole excitations is whether low-lying states are comprised of free charge carriers or excitons. A con- 
ventional approach implies that if the Coulomb interaction is effectively screened and weak, then the electrons and holes are only weakly bound and move essentially independently as free charge-carriers. However, if the Coulomb interaction between electron and hole is strong, excitons are believed to form, i.e. bound particle-hole pairs with strong correlation of their mutual motion.

One of the most popular criteria to discriminate between the states relates to the band gap: states below the charge gap correspond to excitons with binding energy $E_{b}=E_{g}-E$, and states above the charge gap do to free electron-hole pairs. However, this criteria seems to be oversimplified, and the states should be characterized as bound or unbound according to the scaling of the average electron-hole separation with system size. Excitons are entities with small electron-hole separation which remain finite as the system size is increased. By contrast, the average separation between two free charge carriers increases indefinitely with system size. To distinguish bound and unbound electron-hole states one might use the density-density correlation function ${ }^{31}$

$$
C(i, j)=\left\langle\left(\hat{n}_{i}-\left\langle\hat{n}_{i}\right\rangle\right)\left(\hat{n}_{j}-\left\langle\hat{n}_{j}\right\rangle\right)\right\rangle,
$$

which measures a correlation of charge fluctuations on site $i$ to a charge fluctuations on site $j$. A negative value correlates an excess (deficit) of charge with deficit (excess), or electron-hole distribution. In frame of pseudospin approach this correlation function measures the longitudinal $(\| z)$ short-range antiferromagnetic fluctuations

$$
C(i, j)=\left\langle\hat{S}_{i z} \hat{S}_{j z}\right\rangle-\left\langle\hat{S}_{i z}\right\rangle\left\langle\hat{S}_{j z}\right\rangle .
$$

The results of the direct computations for conjugated polymers in frames of the extended Hubbard mode ${ }^{31}$ show for different symmetry sectors that there exist unbound states below the charge gap, and bound states above the charge gap. Thus, the charge gap, often used to define the binding energy of excitons, is not a decisive criterion by which to decide whether a state is bound or unbound.

In practice, many authors consider excitons to consist of real-space configurations with electrons and holes occupying the nearest neighbor sites, while the electrons and holes are separated from each other in the conduction-band states 32 .

The relative energy position and transition intensity of excitons and free unbound electron-hole pairs is an issue of large complexity. For instance, in polydiacetylenes ${ }^{32}$ the large absorption peak at $1.85 \mathrm{eV}$ is attributed to an exciton, since photoconductivity is absent in this energy region. The latter has a threshold at around $2.4 \mathrm{eV}$. Interestingly, that observed photoconductivity band is not visible in the conventional linear (one-photon) absorption spectrum. Because of the strong oscillator strength of the exciton, the conduction band has a weak oscillator strength and is enveloped by the high-energy tail of the exciton peak.

\section{B. Coupling with electromagnetic field}

The electron-hole excitations and optical properties of strongly correlated electron systems is of current both experimental and theoretical interest. In particular, the optical conductivity is of fundamental theoretical interest because the spectral weight at low frequencies seems to be the natural order parameter for the Mott transition. The great body of experimental information regarding the eh-excitations in solids is provided by optical and electron energy loss (EELS) spectroscopy, however, its interpretation depends crucially on the theoretical scheme used. The optical conductivity of different model systems has been studied by approximate mean field calculations, by analysis of integrable 1D models, by exact diagonalization of small systems, and by quantum Monte Carlo techniques. The uncertain quantitative applicability of the analytic mean field calculations, the size limitations of the exact diagonalization and Monte Carlo results and the possibility that the integrable 1D models do not exhibit generic behavior one lead to consider other methods for obtaining information about the optical conductivity. An outgoing beyond the effective Hamiltonian methods with restricted basis which are relevant for description of the lowest energy excitations in the extremely limited range of energies, and the elaboration of effective methods to describe optically excited states is a challenging problem in solid state physics.

Making use of a standard Peierls transformation in hopping terms in (24) we arrive at an effective Hamiltonian for the coupling with electromagnetic field

$$
\begin{gathered}
\hat{t}_{i j} \rightarrow \hat{t}_{i j} e^{i\left(\Phi_{j}-\Phi_{i}\right)}, \\
\left(\Phi_{j}-\Phi_{i}\right)=-\frac{q}{\hbar c} \int_{\mathbf{R}_{i}}^{\mathbf{R}_{j}} \mathbf{A}(\mathbf{r}) d \mathbf{r},
\end{gathered}
$$

where $\mathbf{A}$ is the vector-potential, and integration runs over line binding the $i$ and $j$ sites. For the nearest neighbours one may use the simplified relation

$$
\left(\Phi_{j}-\Phi_{i}\right)=-\frac{q}{\hbar c}\left(\mathbf{A}\left(\mathbf{R}_{i}\right) \cdot \mathbf{R}_{j i}\right)
$$

The current density operator one may represent to be a sum of three terms

$$
\begin{aligned}
& \mathbf{j}^{e e}\left(\mathbf{R}_{i}\right)=\frac{i q}{2 \hbar} \sum_{j} t_{i j} \mathbf{R}_{i j}\left(\hat{a}_{i}^{\dagger} \hat{a}_{j}-\hat{a}_{i} \hat{a}_{j}^{\dagger}\right), \\
& \mathbf{j}^{h h}\left(\mathbf{R}_{i}\right)=\frac{i q}{2 \hbar} \sum_{j} t_{i j} \mathbf{R}_{i j}\left(\hat{b}_{i}^{\dagger} \hat{b}_{j}-\hat{b}_{i} \hat{b}_{j}^{\dagger}\right), \\
& \mathbf{j}^{e h}\left(\mathbf{R}_{i}\right)=\frac{i q}{2 \hbar} \sum_{j} t_{i j} \mathbf{R}_{i j}\left(\hat{a}_{i}^{\dagger} \hat{b}_{j}^{\dagger}-\hat{b}_{i} \hat{a}_{j}\right),
\end{aligned}
$$


where the two first terms describe the electron and hole currents, respectively, while the third term describe the current fluctuations due to eh-pair creation/annihilation.

Standard linear-response theory then yields for optical conductivity $(T=0)$

$$
\operatorname{Re} \sigma(\mathbf{q}, \omega)=(\pi / \omega) \sum_{e}\left|\mu_{g e}(\mathbf{q})\right|^{2} \delta\left(E_{e}-E_{g}-\hbar \omega\right)
$$

where

$$
\mu_{g e}(\mathbf{q})=\left\langle\Psi_{g}\left|j_{\mathbf{q}}\right| \Psi_{e}\right\rangle
$$

is a transition matrix element, and sum runs over all the excited $\Psi_{e}$ states.

The first two current density operators (28)-(29) describe electron and hole intraband transition so that the optical absorption spectra in $M^{0}$ phase are specified only by the latter term (30). In other words, the optical response in $M^{0}$ phase of our model system is determined only through a CT exciton channel.

It should be noted that the electron/hole current operators in (28)-29) can be expressed through a superposition of antisymmetric pseudospin operators:

$$
\left.\left(\hat{S}_{i+} \hat{S}_{j-}-\hat{S}_{i-} \hat{S}_{j+}\right)-\hat{T}_{i+} \hat{T}_{j-}-\hat{T}_{i-} \hat{T}_{j+}\right),
$$

while eh-current fluctuation operator in (30) can be expressed through another superposition of the same antisymmetric pseudospin operators

$$
\left.\left(\hat{S}_{i+} \hat{S}_{j-}-\hat{S}_{i-} \hat{S}_{j+}\right)+\hat{T}_{i+} \hat{T}_{j-}-\hat{T}_{i-} \hat{T}_{j+}\right) .
$$

\section{Charge transfer instability and CT exciton self-trapping}

Electron and hole in a CT exciton in type II $M^{0}$ insulator are strongly coupled both in between and with the lattice. In contrast with conventional wide-band semiconductors where the excitons dissociate easily producing two-component electron-hole gas or plasma, ${ }^{33}$ small CT excitons both free and self-trapped are likely to be stable with regard the eh-dissociation. To illustrate the principal features of CT exciton self-trapping effect we address a simplified two-level model of a two-center MM cluster in which a ground state and a CT state are associated with a pseudospin $1 / 2$ doublet, $|\uparrow\rangle$ and $|\downarrow\rangle$, respectively. In addition, we introduce some configurational coordinate $Q$ associated with a deformation of the cluster, or respective anionic background ${ }^{7}$ Such a model is typical one for so-called (pseudo)Jahn-Teller systems. As a starting point of the model we introduce the effective electron-configurational Hamiltonian as follows

$$
H_{s}=-\Delta \hat{s}_{z}-t \hat{s}_{x}-p Q+\frac{K}{2} Q^{2}-a Q \hat{s}_{z}
$$

where $s_{z}=\frac{1}{2}\left(\begin{array}{ll}1 & 0 \\ 0 & -1\end{array}\right), s_{x}=\frac{1}{2}\left(\begin{array}{ll}0 & 1 \\ 1 & 0\end{array}\right)$ are Pauli matrices, and the first term describes the bare energy splitting of "up" and "down" states with energy gap $\Delta$ (CT energy), while the second term describes the coupling (mixing) between "up" and "down" pseudospin states. In terms of a pseudospin analogy the both parameters may be associated with effective fields. The third and fourth terms in (22) describe the linear and quadratic contributions to the configurational energy. Here, the linear term formally corresponds to the energy of an external configurational strain described by an effective strain parameter $p$, while quadratic term with "elastic" constant $K$ is associated with a conventional harmonic approximation for configurational energy. The last term describes the electron-configurational (vibronic) interaction with $a$ being a electron-configurational coupling constant. Hereafter we make use of dimensionless configurational variable $Q$ therefore all of the model parameters are assigned the energy units. Our model Hamiltonian has the most general form except the simplified form of electronconfigurational coupling where we omit the term $\propto Q \hat{s}_{x}$. In frame of adiabatic approximation the eigenvectors for the Hamiltonian can be written as follows:

$$
\begin{aligned}
& \Psi_{+}(Q)=\cos \alpha|\uparrow\rangle+\sin \alpha|\downarrow\rangle ; \\
& \Psi_{-}(Q)=\sin \alpha|\uparrow\rangle-\cos \alpha|\downarrow\rangle,
\end{aligned}
$$

where

$$
\tan 2 \alpha=\frac{t}{\Delta+a Q}
$$

The corresponding eigenvalues

$$
E_{ \pm}(Q)=\frac{K}{2} Q^{2}-p Q \pm \frac{1}{2}\left[(\Delta+a Q)^{2}+t^{2}\right]^{1 / 2}
$$

define the upper and lower branches of the configurational, or adiabatic potential (AP), respectively. These potential curves describe the energy of $| \pm\rangle$ states as functions of configurational coordinate $Q$. The impact of different model parameters on the shape of AP can be easily understood, if we neglect transfer integral $(t=0)$ and deal with two ideal parabolas describing the configurational energy for "up" and "down" states $|\uparrow, \downarrow\rangle$, respectively. These identical parabolas with minima at $Q_{ \pm}^{(0)}=\left(p \mp \frac{a}{2}\right) / K$ are shifted relative to each other. The shift in configurational coordinate is $\Delta Q=Q_{+}^{(0)}-Q_{-}^{(0)}=$ $a / 2 K$, while the shift in energy is $\Delta E^{(0)}=E_{+}^{(0)}-E_{-}^{(0)}=$ $p a / K+\Delta$, where $E_{ \pm}^{(0)}=E_{ \pm}\left(Q_{ \pm}^{(0)}\right)$ or the energy of the bottoms of respective parabolas. Interestingly, the energy shift is determined both by "mechanic" and "electronic" forces. It is worth noting that the $Q$-shift of the "center of gravity" of AP is determined by the effective strain $p$. The electron-configurational coupling leads to a pulling apart the parabolas for "up" and "down" electronic states. The condition

$$
\left|\Delta E^{(0)}\right|=|p a / K+\Delta|=a^{2} / 2 K
$$




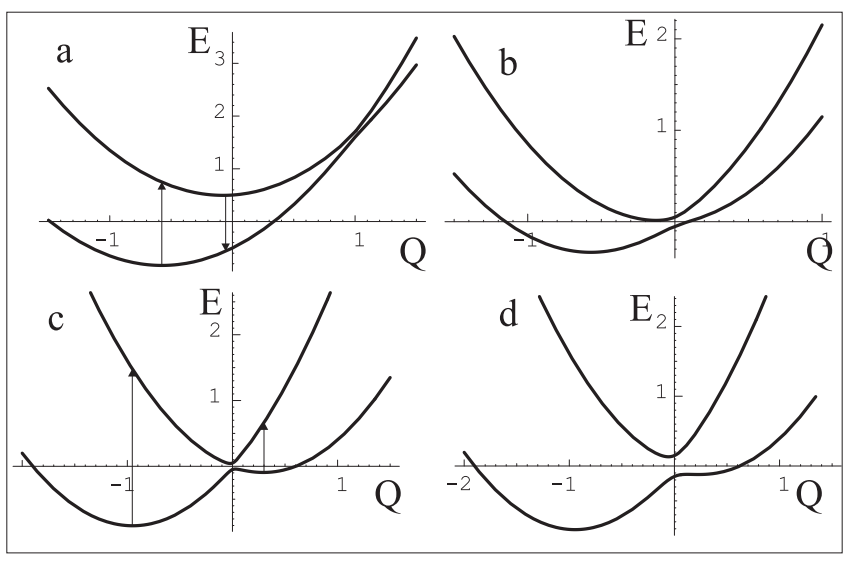

FIG. 3: Typical adiabatic potentials of CT center: a) $\Delta=$ $1.0 ; t=0.1 ; p=-0.65 ; a=1.0 ; K=2.0 ; \mathrm{b}) \Delta=0.0 ; t=$ $0.1 ; p=-0.65 ; a=1.0 ; K=2.0 ;$ c) $\Delta=0.0 ; t=0.1 ; p=$ $-0.65 ; a=2.5 ; K=2.0 ; \mathrm{d}) \Delta=0.0 ; t=0.3 ; p=-0.65 ; a=$ $2.5 ; K=2.0$. Arrows mark CT transitions (see text for details).

defines two specific points, where the minimum of one of the branches crosses another branch, thus specifying the parameters range admissive of a bistability effect. If $E_{+}^{0}>E_{-}\left(Q_{+}^{(0)}\right)$ we arrive at rather conventional situation which is typical for long-lived CT states capable of decaying due to spontaneous Franck-Condon radiative transitions. In opposite case $E_{+}^{0}<E_{-}\left(Q_{+}^{(0)}\right)$ we deal with only radiativeless relaxation channels, and the situation strongly depends on the magnitude of transfer integral $t \neq 0$ which leads to a crucial rearrangement of AP near the crossection point of two branches with a number of novel effects of principal importance. First, we obtain two isolated branches of AP, the upper and lower ones, respectively, with quantum superpositions $\Psi_{ \pm}$which describe unconventional states with a mixed valence of $\mathrm{CuO}_{4}$ plaquettes. An illustrative example of typical adiabatic potentials is shown in Fig.3. Upper branch of AP has a single minimum point $\mathrm{S}$ with approximately "fifty-fifty" composition $|\uparrow, \downarrow\rangle$ of the respective superposition state $\Psi_{+}$.

For lower branch of AP we have either a single minimum point or the two-well structure with two local minimum points (see Fig.3c), leading to a "bistability" effect which is of primary importance for our analysis. Indeed, these two points may be associated with two (meta)stable charge states with and without CT, respectively, which form two candidates to struggle for a ground state.

It is easy to see, that for large values of the transfer integral the system does not manifest bistability, i.e. the lower branch of conformational potential has only a single minimum (see, e.g., Figs.3c,d).

Thus we conclude that all the systems such as copper oxides may be divided to two classes: CT stable systems with the only lower AP branch minimum for a certain charge configuration, and bistable, or CT unstable systems with two lower AP branch minima for two local charge configurations one of which is associated with selftrapped CT excitons resulting from self-consistent charge transfer and electron-lattice relaxation ${ }^{5}$ Moreover, in the latter case we deal with an additional metastable state on the upper AP branch which is characterized by a mixed valence and ionicity and seems to be intermediate one.

Our simple model analysis allows to uncover the relative role of different parameters governing the charge stability in the system. At the same time one should note that actually we deal with more complex multimode problem with a large body of intermediate metastable states which can be seen in luminescence spectra.

\section{Nucleation of EH droplets and phase separation effects in CT unstable $M^{0}$ phase}

The AP bistability in CT unstable insulators points to tempting perspectives of their evolution under either external impact. Metastable CT excitons in the CT unstable $M^{0}$ phase being the disproportionation quanta present candidate "relaxed excited states" to struggle for stability with ground state and the natural nucleation centers for electron-hole liquid phase. What way the CT unstable $M^{0}$ phase can be transformed into novel phase? It seems likely that such a phase transition could be realized due to a mechanism familiar to semiconductors with filled bands such as Ge and Si where given certain conditions one observes a formation of metallic EH-liquid as a result of the exciton decay 33 However, the system of strongly correlated electron $M^{-}$and hole $M^{+}$centers appears to be equivalent to an electron-hole Bose-liquid in contrast with the electron-hole Fermi-liquid in conventional semiconductors. However, the Wannier excitons in the latter wide-band systems dissociate easily producing two-component electron-hole gas or plasma, ${ }^{33}$ while small CT excitons both free and self-trapped are likely to be stable with regard the el-h-dissociation. At the same time, the two-center CT excitons have a very large fluctuating electrical dipole moment $|d| \sim 2 e R_{M M}$ and can be involved into attractive electrostatic dipole-dipole interaction. Namely this is believed to be important incentive to the proliferation of excitons and its clusterization. The CT excitons are proved to attract and form molecules called biexcitons, and more complex clusters where the individuality of the separate exciton is likely to be lost. Moreover, one may assume that like the semiconductors with indirect band gap structure, it is energetically favorable for the system to separate into a low density exciton phase coexisting with the microregions of a high density two-component phase composed of electron $M^{-}$and hole $\mathrm{M}^{+}$centers, or EH droplets. Indeed, the excitons may be considered to be well defined entities only at small content, whereas at large densities their coupling is screened and their overlap becomes so considerable that they loose individuality and we come to the system of electron $M^{-}$ and hole $M^{+}$centers, which form a electron-hole Bose liquid. An increase of injected excitons in this case merely 
increases the size of the EH droplets, without changing the free exciton density.

Homogeneous nucleation implies the spontaneous formation of EH droplets due to the thermodynamic fluctuations in exciton gas. Generally speaking, such a state with a nonzero volume fraction of $\mathrm{EH}$ droplets and the spontaneous breaking of translational symmetry can be stable in nominally pure insulating crystal. However, the level of intrinsic non-stoihiometry in 3d oxides is significant (one charged defect every 100-1000 molecular units is common). The charged defect produces random electric field, which can be very large (up to $10^{8} \mathrm{Vcm}^{-1}$ ) thus promoting the condensation of CT excitons and the inhomogeneous nucleation of EH droplets. Deviation from the neutrality implies the existence of additional electron, or hole centers that can be the natural centers for the inhomogeneous nucleation of the EH droplets. Such droplets are believed to provide the more effective screening of the electrostatic repulsion for additional electron/hole centers, than the parent insulating phase. As a result, the electron/hole injection to the insulating $M^{0}$ phase due to a nonisovalent substitution as in $\mathrm{La}_{2-x} \mathrm{Sr}_{x} \mathrm{CuO}_{4}$, $\mathrm{Nd}_{2-x} \mathrm{Ce}_{x} \mathrm{CuO}_{4}$, or change in oxygen stoihiometry as in $\mathrm{YBa}_{2} \mathrm{Cu}_{3} \mathrm{O}_{6+x}, \mathrm{La}_{2} \mathrm{CuO}_{4-\delta}, \mathrm{La}_{2} \mathrm{Cu}_{1-x} \mathrm{Li}_{x} \mathrm{O}_{4}$, or fieldeffect is believed to shift the phase equilibrium from the insulating state to the unconventional electron-hole Bose liquid, or in other words induce the insulator-to-EHBL phase transition.

The optimal way to the nucleation of $\mathrm{EH}$ droplets in parent system like $\mathrm{La}_{2} \mathrm{CuO}_{4}, \mathrm{YBa}_{2} \mathrm{Cu}_{3} \mathrm{O}_{6}$ is to create charge inhomogeneity by nonisovalent chemical substitution in $\mathrm{CuO}_{2}$ planes or in "out-of-plane stuff", including interstitial atoms and vacancies. This process results in an increase of the energy of the parent phase and creates proper conditions for its competing with others phases capable to provide an effective screening of the charge inhomogeneity potential. The strongly degenerate system of electron and hole centers in $\mathrm{EH}$ droplet is one of the most preferable ones for this purpose. At the beginning (nucleation regime) an EH droplet nucleates as a nanoscopic cluster composed of several number of neighboring electron and hole centers pinned by disorder potential. Charged defects supporting the EH droplet nucleation promote the formation of metastable ("superheated") clusters of parent phase. It is clear that such a situation does not exclude the self-doping with the formation of a self-organized collective charge-inhomogeneous state in systems which are near the charge instability.

EH droplets can manifest itself remarkably in various properties of the $3 \mathrm{~d}$ oxides even at small volume fraction, or in a "pseudo-impurity regime". Insulators in a PS regime should be considered as phase inhomogeneous systems with, in general, thermo-activated mobility of the inter-phase boundaries. On the one hand, main features of this "pseudo-impurity regime" would be determined by the partial intrinsic contributions of the appropriate phase components with possible limitations imposed by the finite size effects. On the other hand, the real prop- erties will be determined by the peculiar geometrical factors such as a volume fraction, average size of droplets and its dispersion, the shape and possible texture of the droplets, the geometrical relaxation rates. These factors are tightly coupled, especially near phase transitions for either phase (long range antiferromagnetic ordering for the parent phase, the charge ordering and other phase transformations for the EH droplets) accompanied by the variation in a relative volume fraction. Numerous examples of the unconventional behavior of the $3 \mathrm{~d}$ oxides in the pseudo-impurity regime could be easily explained with taking into account the inter-phase boundary effects (coercitivity, the mobility threshold, non-ohmic conductivity, oscillations, relaxation etc.) and corresponding characteristic quantities. Under increasing doping the "pseudo-impurity regime" with a relatively small volume fraction of EH droplets (nanoscopic phase separation) can gradually transform into a macro- (chemical) "phaseseparation regime" with a sizeable volume fraction of $\mathrm{EH}$ droplets, and finally to a new EH liquid phase. Phase separation is now widely discussed as an important phenomenon accompanying the high- $\mathrm{T}_{c}$ superconductivity.

Our scenario can readily explain photo-induced effects in pseudo-impurity phase ${ }^{34}$ Indeed, the illumination of a material with light leads to the generation of eh-pairs that will proliferate and grow up to be a novel nonequilibrium EH droplet or simply to be trapped in either EH droplet with the rise in its volume fraction. The excitation energy appears to be lower when exciton is closer to the EH droplet. Therefore once the excitation transfer is finite, the optical excitation is attracted to the nearest neighbour of the EH clusters so that this cluster expands effectively under the light irradiation. In other words, the photoexcitation would result in an increase of the $\mathrm{EH}$ droplet volume fraction, that is why its effect in optical response resembles that of chemical doping. After switching off the light the droplet phase would relax to the thermodynamically stable state. Furthermore, such a simple model can immediately explain the persistent photoconductivity (PPC) phenomena, found in insulating $\mathrm{YBaCuO}$ system, 35 where the oxygen reodering provides the mechanism of a long-time stability for the $\mathrm{EH}$ droplets. In PPC phenomena, an illumination of a material with light leads to a long-lived photoconductive state. During the illumination of underdoped $\mathrm{YBaCuO}$ near the insulator-metal transition, the material may even become superconducting.

\section{ELECTRON-HOLE BOSE LIQUID}

Hereafter we would like to address the bosonic nature and some properties of the mixed-valence binary (disproportionated) $M^{ \pm}$-phase termed as electron-hole Bose liquid (EHBL) in more details. For such a system, the pseudo-spin Hamiltonian (22) can be mapped onto the Hamiltonian of hard-core Bose gas on a lattice (BoseHubbard model) which standard form can be written out 
as follows: $:^{36,37,38}$

$$
H_{B G}=-\sum_{i>j} t_{i j}\left(B_{i}^{\dagger} B_{j}+B_{j}^{\dagger} B_{i}\right)+\sum_{i>j} V_{i j} N_{i} N_{j}-\mu \sum_{i} N_{i}
$$

where $N_{i}=B_{i}^{\dagger} B_{i}, \mu$ is a chemical potential derived from the condition of fixed full number of bosons $N_{l}=\sum_{i=1}^{N}\left\langle N_{i}\right\rangle$, or concentration $n=N_{l} / N \in[0,1]$. The $t_{i j}$ denotes an effective transfer integral, $V_{i j}$ is an intersite interaction between the bosons. Here $B^{\dagger}(B)$ are the Pauli creation (annihilation) operators which are Bose-like commuting for different sites $\left[B_{i}, B_{j}^{\dagger}\right]=0$, for $i \neq j$, and for the same site $B_{i}^{2}=\left(B_{i}^{\dagger}\right)^{2}=0,\left[B_{i}, B_{i}^{\dagger}\right]=1-2 N_{i}, N_{i}=B_{i}^{\dagger} B_{i} ; N$ is a full number of sites.

The disproportionated phase as well as the lattice hard-core Bose-gas is equivalent to a system of spins $s=1 / 2$ exposed to an external magnetic field in the $z$ direction. Indeed, the charge $(e, h)$, or $M^{\mp}$-doublet, that is two different valence states of $M$-centers, one might associate with two possible states of the charge pseudo$\operatorname{spin}$ (isospin) $s=\frac{1}{2}:\left|+\frac{1}{2}\right\rangle$ and $\left|-\frac{1}{2}\right\rangle$ for electron $M^{-}$ and hole $M^{+}$centers, respectively. Then the effective Hamiltonian can be written as follows $\frac{36.37 .38}{}$

$$
H_{B G}=\sum_{i>j} J_{i j}^{x y}\left(s_{i}^{+} s_{j}^{-}+s_{j}^{+} s_{i}^{-}\right)+\sum_{i>j} J_{i j}^{z} s_{i}^{z} s_{j}^{z}-h \sum_{i} s_{i}^{z},
$$

where $J_{i j}^{x y}=2 t_{i j}, J_{i j}^{z}=V_{i j}, h=\mu-\sum_{j(j \neq i)} V_{i j}, s^{-}=$ $\frac{1}{\sqrt{2}} B, s^{+}=-\frac{1}{\sqrt{2}} B^{\dagger}, s^{z}=-\frac{1}{2}+B_{i}^{\dagger} B_{i}, s^{ \pm}=\mp \frac{1}{\sqrt{2}}\left(s^{x} \pm \imath s^{y}\right)$. Below we make use of conventional two-sublattice approach. For the description of the pseudospin ordering to be more physically clear one may introduce two vectors, the ferromagnetic and antiferromagnetic ones:

$$
\mathbf{m}=\frac{1}{2 s}\left(\left\langle\mathbf{s}_{1}\right\rangle+\left\langle\mathbf{s}_{2}\right\rangle\right) ; \mathbf{l}=\frac{1}{2 s}\left(\left\langle\mathbf{s}_{1}\right\rangle-\left\langle\mathbf{s}_{2}\right\rangle\right) ; \mathbf{m}^{2}+\mathbf{l}^{2}=1,
$$

where $\mathbf{m} \cdot \mathbf{l}=0$. The hard-core boson system in a twosublattice approximation is described by two diagonal order parameters $l_{z}, m_{z}$ and two complex off-diagonal order parameters: $m_{ \pm}=\mp \frac{1}{\sqrt{2}}\left(m_{x} \pm \imath m_{y}\right)$ and $l_{ \pm}=$ $\mp \frac{1}{\sqrt{2}}\left(l_{x} \pm \imath l_{y}\right)$. The complex superfluid order parameter $\Psi(\mathbf{r})=|\Psi(\mathbf{r})| \exp -\imath \phi$ is determined by the in-plane components of ferromagnetic vector: $\Psi(\mathbf{r})=\frac{1}{2}\left\langle\left(\hat{B}_{1}+\hat{B}_{2}\right)\right\rangle=$ $s m_{-}=s m_{\perp} \exp -\imath \phi, m_{\perp}$ being the length of the in-plane component of ferromagnetic vector. So, for a local condensate density we get $n_{s}=s^{2} m_{\perp}^{2}$. It is of interest to note that in fact all the conventional uniform $T=0$ states with nonzero $\Psi(\mathbf{r})$ imply simultaneous long-range order both for modulus $|\Psi(\mathbf{r})|$ and phase $\phi$. The in-plane components of antiferromagnetic vector $l_{ \pm}$describe a staggered off-diagonal order.

The model exhibits many fascinating quantum phases and phase transitions. Early investigations predict at $T=0$ charge order $(\mathrm{CO})$, Bose superfluid (BS) and mixed $(\mathrm{BS}+\mathrm{CO})$ supersolid uniform phases with an Ising-type melting transition (CO-NO) and KosterlitzThouless-type (BS-NO) phase transitions to a nonordered normal fluid (NO) in $2 \mathrm{D}$ systems

\section{TOPOLOGICAL CHARGE FLUCTUATIONS IN MODEL MIXED-VALENCE SYSTEM}

Above we focused on the homogeneous phase states of the mixed-valence system. Main short-length scale charge fluctuations in $M^{0}$ and $M^{ \pm}$systems are associated with a thermal exciton creation, or annihilation due to a reaction: $\left(M^{0}-M^{0}\right) \leftrightarrow\left(M^{+}-M^{-}\right)$. Amongst the long-length scale charge fluctuations in a model system we would like to address the topological defects in quasi-2D systems such as cuprates, in particular, different bubble-like entities like skyrmions, or another out-ofplane vortices. Namely these one can play the main role in a nucleation of unconventional charge phases.

\section{A. Topological defects in $M^{0}$-phase}

The most interesting situation concerns the charge fluctuations in the conceptually simple monovalent $M^{0}$ phase which seems to be a representative of traditional insulating oxides. From the viewpoint of the $\mathbf{a}, \mathbf{b}$-vector field formalism the $M^{0}$ system resembles, in a sense, the easy-axis (anti)ferromagnet. Indeed, the phase is specified by a simple uniform arrangement of $\mathbf{a}$ and $\mathbf{b}$ vectors parallel to $z$-axis: a $\|\mathbf{b}\| O_{z}$, and its energy does not depend on the sense of these vectors. The analogy allows us to make use of simple physically clear pictures of (anti)ferromagnetic domain structures.

Below we'll address two types of domain walls in $M^{0}$ phase. The first would illustrate the long-scale fluctuation which conserves the mean on-site valence: in other words, $\left\langle S_{z}\right\rangle=0$. The second would provide the example of a domain wall with a checkerboard charge ordering in its center.

First of all, let note that instead of two parallel vectors $\mathbf{a}$ and $\mathbf{b}$ given the normalization condition, the $M^{0}$-phase can be described by a unified vector $\mathbf{n}: \mathbf{a}=\alpha \mathbf{n}, \mathbf{b}=\beta \mathbf{n}$, and $\alpha+i \beta=\exp (i \kappa), \kappa \in R$. Hereafter, we denote $\mathbf{n}=\mathbf{n}\{\sin \theta \cos \phi, \sin \theta \sin \phi, \cos \theta\}$. Moreover, we may introduce a multitude of phases which differ only by the orientation of the unit vector $\mathbf{n}$, or $\mathbf{n}$-phases. Such phases may be considered to be the solutions of a purely biquadratic pseudo-spin Hamiltonian ${ }^{30}$

$$
\hat{H}_{b q}=-J_{2} \sum_{i, \eta} \sum_{k \geq j}^{3}\left(\left\{\hat{S}_{k} \hat{S}_{j}\right\}_{i}\left\{\hat{S}_{k} \hat{S}_{j}\right\}_{i+\eta}\right),
$$

which can be obtained from (19) given the only nonzero "exchange" parameters $J_{k k}$ with $k=4 \div 8$. The quantum 
Hamiltonian can be mapped onto classical Hamiltonian ${ }^{30}$

$$
H_{b q}=2 J_{2}|\mathbf{n}|^{2} \int d^{2} \mathbf{r}\left[\sum_{i=1}^{3}\left(\nabla \mathbf{n}_{\mathbf{i}}\right)^{2}\right]
$$

The constant zero value of the mean on-site valence $\left\langle S_{z}\right\rangle=0$ is the common property of the $\mathbf{n}$-phases. Moreover, the mean value of all the pseudo-spin components turns into zero: $\langle\mathbf{S}\rangle=0$. Let address the ferromagnetic n-phase with $180^{\circ}$ domain walls which separate two actually equivalent $M^{0}$-domains with opposite direction of $\mathbf{n}$ vectors. The picture differs from that of conventional ferromagnets where $180^{\circ}$ domain wall separates the domains with the opposite direction of magnetization. In the center of such a $B S$-type domain wall we may deal with a superfluid BS phase $\mathbf{n} \perp O_{z}$.

In $2 \mathrm{D}$ systems such as cuprates it appears possible to form skyrmion-like topological defects like bubbles ${ }^{30}$ The skyrmion spin texture looks like a bubble domain in ferromagnet and consists of a vortex-like arrangement of the in-plane components of spin with the $z$-component reversed in the centre of the skyrmion and gradually increasing to match the homogeneous background at infinity. The spin distribution within a classical BelavinPolyakov (BP) skyrmion is given as follows 39

$$
\phi=q \varphi+\varphi_{0} ; \quad \cos \theta=\frac{r^{2 q}-\lambda^{2 q}}{r^{2 q}+\lambda^{2 q}}
$$

or for the winding number $q=1$

$$
n_{x}=\frac{2 r \lambda}{r^{2}+\lambda^{2}} \cos \phi ; n_{y}=\frac{2 r \lambda}{r^{2}+\lambda^{2}} \sin \phi ; n_{z}=\frac{r^{2}-\lambda^{2}}{r^{2}+\lambda^{2}} .
$$

Skyrmions are characterized by the magnitude and sign of its topological charge, by its size (radius) $\lambda$, and by the global orientation of the spin, or $\mathrm{U}(1)$ order parameter $\varphi_{0}$. The scale invariance of skyrmionic solution reflects in that its energy is proportional to winding number and does not depend on radius, and global phase $\varphi_{0}$. An interesting example of topological inhomogeneity is provided by a multi-center $\mathrm{BP}$ skyrmion ${ }^{39}$ which energy does not depend on the position of the centers. The latter are believed to be addressed as an additional degree of freedom, or positional order parameter.

The classical Hamiltonian (41) has skyrmionic solutions, but instead of the spin distribution in conventional BP skyrmion ${ }^{39}$ we deal with a zero spin, but a non-zero distribution of five spin-quadrupole moments $\left\langle\Lambda^{(4,5,6,7,8)}\right\rangle$, or $\left\langle\left\{S_{i} S_{j}\right\}\right\rangle$ which in turn are determined by the skyrmionic texture of the $\mathbf{n}$ vector:

$$
\begin{gathered}
\left\langle\left(S_{x}^{2}+S_{y}^{2}\right)\right\rangle=1+\cos ^{2} \theta ;\left\langle S_{z}^{2}\right\rangle=\sin ^{2} \theta ;\left\langle\left(S_{x}^{2}-S_{y}^{2}\right)\right\rangle=-\sin ^{2} \theta \cos 2\left(q \varphi+\varphi_{0}\right) ; \\
\left\langle\left\{S_{x} S_{y}\right\}\right\rangle=-\sin ^{2} \theta \sin 2\left(q \varphi+\varphi_{0}\right) ;\left\langle\left\{S_{x} S_{z}\right\}\right\rangle=-\sin 2 \theta \cos \left(q \varphi+\varphi_{0}\right) ;\left\langle\left\{S_{y} S_{z}\right\}\right\rangle=-\sin 2 \theta \sin \left(q \varphi+\varphi_{0}\right) .
\end{gathered}
$$

Thus we arrive at the ring-shaped distribution of the effective ionicity $\left\langle S_{z}^{2}\right\rangle$ with the maximal value of 1 ( $M^{ \pm}$-phase) at $r=\lambda$, and the minimal value of 0 at the ring center $r=0$ and far outside $r \rightarrow \infty\left(M^{0}\right.$ phase). The "bosonic" off-diagonal complex order parameter $\left\langle V_{ \pm 2}^{2}\right\rangle \propto\left\langle S_{ \pm}^{2}\right\rangle \propto \sin ^{2} \theta \exp \left( \pm 2\left(q \varphi+\varphi_{0}\right)\right)$ has a similar $r$-dependence, while the "fermionic" off-diagonal complex order parameter $\left\langle V_{ \pm 1}^{2}\right\rangle \propto \sin 2 \theta \exp \left( \pm\left(q \varphi+\varphi_{0}\right)\right)$ turns into zero both at the ring center $r=0$, far outside $r \rightarrow \infty\left(M^{0}\right.$-phase $)$, and at $r=\lambda$, or everywhere, where the ionicity has a strictly definite value.

Despite these skyrmions are derived from the toy model, they yield very instructive information as regards the probable spin texture of real solitons with BS-type domain walls and "superconductive" fluctuations in $M^{0}$ phase.

The CO-type domain walls with a nonzero mean onsite valence given the total $\sum_{i}\left\langle S_{z}\right\rangle=0$ can be obtained, in common, in a two-sublattice approximation with non-collinear $\mathbf{a}$ and $\mathbf{b}$ vectors. Let assume the $M^{0}$ - phase to be divided onto two sublattices, $\mathrm{A}$ and $\mathrm{B}$, with $\mathbf{a}_{A}=\mathbf{b}_{A}=\mathbf{a}_{B}=\mathbf{b}_{B}$ and $\mathbf{l}_{A}=\mathbf{l}_{B}=0$. Then the CO-type domain wall may be represented by a gradual spatial non-equivalent rotation of $\mathbf{a}$ and $\mathbf{b}$ vectors in $\mathrm{A}$ and $\mathrm{B}$ sublattices providing the nonzero magnitude of $l_{z}$ components given $l_{z A}=-l_{z B}$ with its maximum in the center of the domain wall.

\section{B. Topological phase separation in 2D EH Bose liquid away from half-filling}

One of the fundamental hot debated problems in bosonic physics concerns the evolution of the charge ordered $(\mathrm{CO})$ ground state of $2 \mathrm{D}$ hard-core $\mathrm{BH}$ model (hc-BH) with a doping away from half-filling. Numerous model studies steadily confirmed the emergence of "supersolid" phases with simultaneous diagonal and offdiagonal long range order while Penrose and Onsager 40 were the first showing as early as 1956 that supersolid 
phases do not occur. The most recent quantum MonteCarlo (QMC) simulations ${ }^{41.42 .43}$ found two significant features of the 2D Bose-Hubbard model with a screened Coulomb repulsion: the absence of supersolid phase at half-filling, and a growing tendency to phase separation $(\mathrm{CO}+\mathrm{BS})$ upon doping away from half-filling. Moreover, Batrouni and Scalettar ${ }^{41}$ studied quantum phase transitions in the ground state of the 2D hard-core boson Hubbard Hamiltonian and have shown numerically that, contrary to the generally held belief, the most commonly discussed "checkerboard" supersolid is thermodynamically unstable and phase separates into solid and superfluid phases. The physics of the $\mathrm{CO}+\mathrm{BS}$ phase separation in Bose-Hubbard model is associated with a rapid increase of the energy of a homogeneous CO state with doping away from half-filling due to a large "pseudo-spin-flip" energy cost. Hence, it appears to be energetically more favorable to "extract" extra bosons (holes) from the CO state and arrange them into finite clusters with a relatively small number of particles. Such a droplet scenario is believed to minimize the long-range Coulomb repulsion.

Magnetic analogy allows us to make unambiguous predictions as regards the doping of $\mathrm{BH}$ system away from half-filling. Indeed, the boson/hole doping of checkerboard $\mathrm{CO}$ phase corresponds to the magnetization of an antiferromagnet in $z$-direction. In the uniform easy-axis $l_{z}$-phase of anisotropic antiferromagnet the local spinflip energy cost is rather big. In other words, the energy cost for boson/hole doping into checkerboard CO phase appears to be big one due to a large contribution of boson-boson repulsion. However, the magnetization of the anisotropic antiferromagnet in an easy axis direction may proceed as a first order phase transition with a "topological phase separation" due to the existence of antiphase domains. The antiphase domain walls provide the natural nucleation centers for a spin-flop phase having enhanced magnetic susceptibility as compared with small if any longitudinal susceptibility thus providing the advantage of the field energy. Namely domain walls would specify the inhomogeneous magnetization pattern for such an anisotropic easy-axis antiferromagnet in relatively weak external magnetic field. As concerns the domain type in quasi-two-dimensional antiferromagnet one should emphasize the specific role played by the cylindrical, or bubble domains which have finite energy and size. These topological solitons have the vortex-like inplane spin structure and resemble classical, or BelavinPolyakov skyrmions ${ }^{39}$ Although some questions were not completely clarified and remain open until now, the classical and quantum skyrmion-like topological defects are believed to be a genuine element of essential physics both of ferro- and antiferromagnetic 2D easy-axis systems. The magnetic analogy seems to be a little bit naive, however, it catches the essential physics of doping the hc-BH system. As regards the checkerboard CO phase of such a system, namely a finite energy skyrmion-like bubble domain ${ }^{44.45}$ seems to be the most preferable candidate for the domain with antiphase domain wall providing the natural reservoir for extra bosons. The skyrmion spin texture consists of a vortex-like arrangement of the inplane components of ferromagnetic $\mathbf{m}$ vector with the $l_{z^{-}}$ component reversed in the centre of the skyrmion and gradually increasing to match the homogeneous background at infinity.

Recently 45 it was shown that the doping, or deviation from half-filling in 2D EH Bose liquid is accompanied by the formation of multi-center topological defect such as charge order (CO) bubble domain(s) with Bose superfluid (BS) and extra bosons both localized in domain wall(s), or a topological $\mathrm{CO}+\mathrm{BS}$ phase separation, rather than an uniform mixed $\mathrm{CO}+\mathrm{BS}$ supersolid phase.

The most probable possibility is that every bubble accumulates one or two particles. Then, the number of such entities in a multigranular texture nucleated with doping has to nearly linearly depend on the doping. Generally speaking, each individual bubble may be characterized by its position, nanoscale size, and the orientation of $\mathrm{U}(1)$ degree of freedom. In contrast with the uniform states the phase of the superfluid order parameter for a bubble is assumed to be unordered. In the long-wavelength limit the off-diagonal ordering can be described by an effective Hamiltonian in terms of $\mathrm{U}(1)$ (phase) degree of freedom associated with each bubble. Such a Hamiltonian contains a repulsive, long-range Coulomb part and a short-range contribution related to the phase degree of freedom. The latter term can be written out in the standard for the $X Y$ model form of a so-called Josephson coupling 44.46 .47

$$
H_{J}=-\sum_{\langle i, j\rangle} J_{i j} \cos \left(\varphi_{i}-\varphi_{j}\right)
$$

where $\varphi_{i}, \varphi_{j}$ are global phases for micrograins centered at points $i, j$, respectively, $J_{i j}$ Josephson coupling parameter. Namely the Josephson coupling gives rise to the long-range ordering of the phase of the superfluid order parameter in such a multi-center texture. Such a Hamiltonian represents a starting point for the analysis of disordered superconductors, granular superconductivity, insulator-superconductor transition with $\langle i, j\rangle$ array of superconducting islands with phases $\varphi_{i}, \varphi_{j}$. To account for Coulomb interaction and allow for quantum corrections we should introduce into effective Hamiltonian the charging energy $\underline{47}$

$$
H_{c h}=-\frac{1}{2} q^{2} \sum_{i, j} n_{i}\left(C^{-1}\right)_{i j} n_{j},
$$

where $n_{i}$ is a number operator for particles bound in $i$ th micrograin; it is quantum-mechanically conjugated to $\varphi: n_{i}=-i \partial / \partial \varphi_{i},\left(C^{-1}\right)_{i j}$ stands for the capacitance matrix, $q$ for a particle charge.

Such a system appears to reveal a tremendously rich quantum-critical structure $\stackrel{48.49}{=}$ In the absence of disorder, the $T=0$ phase diagram of the multi-bubble system 
implies either triangular, or square crystalline arrangements with possible melting transition to a liquid. It should be noted that analogy with charged $2 D$ Coulomb gas implies the Wigner crystallization of multi-bubble system with Wigner crystal (WC) to Wigner liquid melting transition, respectively. Naturally, that the additional degrees of freedom for a bubble provide a richer physics of such lattices. For a system to be an insulator, disorder is required, which pins the multigranular system and also causes the crystalline order to have a finite correlation length. Traditional approach to a Wigner crystallization implies the formation of a $\mathrm{WC}$ for densities lower than a critical density, when the Coulomb energy dominates over the kinetic energy. The effect of quantum fluctuations leads to a (quantum) melting of the solid at high densities, or at a critical lattice spacing. The critical properties of a two-dimensional lattice without any internal degree of freedom are successfully described applying the BKT (Berezinsky-Kosterlitz-Thowless) theory to dislocations and disclinations of the lattice, and proceeds in two steps. The first implies the transition to a liquid-crystal phase with a short-range translational order, the second does the transition to isotropic liquid. For such a system provided the bubble positions fixed at all temperatures, the long-wave-length physics would be described by an (anti)ferromagnetic $X Y$ model with expectable BKT transition and gapless $X Y$ spin-wave mode.

The low temperature physics in a multi-bubble system is governed by an interplay of two BKT transitions, for the $U(1)$ phase and positional degrees of freedom, respectively ${ }^{49}$ Dislocations lead to a mismatch in the $\mathrm{U}(1)$ degree of freedom, which makes the dislocations bind fractional vortices and lead to a coupling of translational and phase excitations. Both BKT temperatures either coincide (square lattice) or the melting one is higher (triangular lattice) $\underline{\underline{49}}$

Quantum fluctuations can substantially affect these results. Quantum melting can destroy U(1) order at sufficiently low densities where the Josephson coupling becomes exponentially small. Similar situation is expected to take place in the vicinity of structural transitions in a multigranular crystal. With increasing the micrograin density the quantum effects result in a significant lowering of the melting temperature as compared with classical square-root dependence. The resulting melting temperature can reveal an oscilating behavior as a function of particle density with zeros at the critical (magic) densities associated with structural phase transitions.

In terms of our model, the positional order corresponds to an incommensurate charge density wave, while the $\mathrm{U}(1)$ order does to a superconductivity. In other words, we arrive at a subtle interplay between two orders. The superconducting state evolves from a charge order with $T_{C} \leq T_{m}$, where $T_{m}$ is the temperature of a melting transition which could be termed as a temperature of the opening of the insulating gap (pseudo-gap!?).

The normal modes of a dilute multi-bubble system include the pseudo-spin waves propagating in-between the bubbles, the positional fluctuations, or quasi-phonon modes, which are gapless in a pure system, but gapped when the lattice is pinned, and, finally, fluctuations in the $\mathrm{U}(1)$ order parameter.

The orientational fluctuations of the multi-bubble system are governed by the gapless $X Y$ model ${ }^{48}$ The relevant model description is most familiar as an effective theory of the Josephson junction array. An important feature of the model is that it displays a quantum-critical point.

The low-energy collective excitations of a multi-bubble liquid includes an usual longitudinal acoustic phononlike branch. The liquid crystal phases differ from the isotropic liquid in that they have massive topological excitations, i.e., the disclinations. One should note that the liquids do not support transverse modes, these could survive in a liquid state only as overdamped modes. So that it is reasonable to assume that solidification of the bubble lattice would be accompanied by a stabilization of transverse phonon-like modes with its sharpening below melting transition. In other words, an instability of transverse phonon-like modes signals the onset of melting. The phonon-like modes in the bubble crystal have much in common with usual phonon modes, however, due to electronic nature these can hardly be detected if any by inelastic neutron scattering.

A generic property of the positionally ordered bubble configuration is the sliding mode which is usually pinned by the disorder. The depinning of sliding mode(s) can be detected in a low-frequency and low-temperature optical response.

\section{IMPLICATIONS FOR STRONGLY CORRELATED OXIDES}

In this Section we suggest some speculations around an unconventional scenario of the essential physics of cuprates and manganites that implies their instability with regard to the self-trapping of charge transfer excitons and the formation of electron-hole Bose liquid.

\section{A. Cuprates}

The origin of high- $\mathrm{T}_{c}$ superconductivity is presently still a matter of great controversy. The unconventional behavior of cuprates strongly differs from that of ordinary metals and merely resembles that of doped semiconductor. Moreover, the history of high $\mathrm{T}_{c}$ 's itself shows that we deal with a transformation of particularly insulating state in which the electron correlations govern the essential physics.

The copper oxides start out life as insulators in contrast with BCS superconductors being conventional metals. It is impossible to understand the behavior of the doped cuprates and, in particular, the origin of HTSC 
unless the nature of the doped-insulating state is incorporated into the theory. The problem of a doped insulator is sure much more complicated than it is implied in oversimplified approaches such as an effective $t$ - $J$-model when the situation resembles that of "throwing the baby out of the bathwater".

In a case of cuprates we deal with systems which conventional ground state seems to be unstable with regard to the transformation into a new phase state with a variety of unusual properties.

In our view, the essential physics of the doped cuprates, as well as other strongly correlated oxides, is driven by a self-trapping of the CT excitons, both one-center, and two-center $\stackrel{18.50}{ }$ Such excitons are the result of selfconsistent charge transfer and lattice distortion with the appearance of the "negative- $U$ " effect. 4.5 The two-center excitons are associated with $\mathrm{CT}$ transitions between two $\mathrm{CuO}_{4}$ plaquettes, and may be considered as quanta of the disproportionation reaction

$$
\mathrm{CuO}_{4}^{6-}+\mathrm{CuO}_{4}^{6-} \rightarrow \mathrm{CuO}_{4}^{7-}+\mathrm{CuO}_{4}^{5-}
$$

with the creation of electron $\mathrm{CuO}_{4}^{7-}$ and hole $\mathrm{CuO}_{4}^{5-}$ centers. Thus, three types of $\mathrm{CuO}_{4}$ centers $\mathrm{CuO}_{4}^{5,6,7-}$ should be considered on equal footing. In this connection we would like to draw special attention to the lattice polarization and relaxation effects that are of primary importance both for the formation of CT exciton itself and its self-trapping. It should be noted that the photo-excited electron-hole pairs or excitons are stabilized into self-trapped excitons (STE) accompanied with lattice relaxation within several pico-seconds ${ }^{11}$

In contrast with $\mathrm{BaBiO}_{3}$ system where we deal with a spontaneous generation of self-trapped CT excitons in the ground state, the parent insulating cuprates are believed to be near excitonic instability when the selftrapped CT excitons form the candidate relaxed excited states to struggle with the conventional ground state ${ }^{11}$ In other words, the lattice relaxed CT excited state should be treated on an equal footing with the ground state. If the interaction between STE were attractive and so large that the cohesive energy $W_{1}$ per one STE exceeds the energy $E_{R}$ of one STE, the STE's and/or its clusters will be spontaneously generated everywhere without any optical excitation, and be condensed to form a new electronic state on a new lattice structure 11

The minimal energy cost of the optically excited disproportionation or electron-hole formation in insulating cuprates is $2.0 \div 2.5 \mathrm{eV}: \frac{18.50}{}$ Interestingly, that this relatively small value of the optical gap was nevertheless used by Goodenough 51 as argument against the "negativeU" disproportionation reaction $2 \mathrm{Cu}(\mathrm{II})=\mathrm{Cu}(\mathrm{III})+$ $\mathrm{Cu}(\mathrm{I})$, or more correctly $2\left[\mathrm{CuO}_{4}^{6-}\right]=\left[\mathrm{CuO}_{4}^{5-}\right]+\left[\mathrm{CuO}_{4}^{7-}\right]$ in cuprates. However, the question arises, what is the energy cost for the thermal excitation of such a disproportionation? In other words, what is the energy of selftrapped two-center CT exciton? It is this quantity rather than its optical counterpart defines the activation energy for such a reaction. The question is of primary importance for the self-trapping scenario. The answer implies first of all the knowledge of electronic and ionic polarization energies for electron and hole. The polarization effects with its typical energy scale of the order of several $\mathrm{eV}$ appear to be of primary importance when one considers different charge fluctuations in insulators.

Several general theories of self-trapping have been proposed starting from works by Landau, Pekar and Toyozawa, however, the stages of the ST process and detailed atomistic and electronic structure of ST-excitons and ST-holes are still unclear even in rather simple and well-studied systems such as alkali-metal halides.

As regards the STE in cuprates, we have some straightforward experimental indications. A key characteristic of the STE is its luminescence: STE are short-lived luminescent states of excited crystals. The observation of photoluminescence (PL) near $2.0 \div 2.4 \mathrm{eV}$ in $\mathrm{La}_{2} \mathrm{CuO}_{4}, \frac{52.53}{1}$ near 1.3 and $2.4 \mathrm{eV}$ in $\mathrm{YBa}_{2} \mathrm{Cu}_{3} \mathrm{O}_{6}, \frac{52.54 .55}{}$ near 1.78, 1.95, $2.06 \mathrm{eV}$ in $\mathrm{PrBa}_{2} \mathrm{Cu}_{3} \mathrm{O}_{6}{ }^{52}$ is a direct evidence of strongly localized long-lived states related to self-trapped excitons or their derivatives. The nearinfrared photoluminescence was observed in many insulating cuprates 52.54 .56 To the best of our knowledge the most detailed PL study was performed by Denisov et $a l^{54}$ in $\mathrm{YBa}_{2} \mathrm{Cu}_{3} \mathrm{O}_{6+x}$ in the spectral range $0.7 \div 1.4 \mathrm{eV}$. The low-temperature $\mathrm{PL}$ in $\mathrm{YBa}_{2} \mathrm{Cu}_{3} \mathrm{O}_{6}$ consists of three peaks at $0.87,1.07$, and $\sim 1.4 \mathrm{eV}$, respectively. The PL intensity is much stronger at small doping level. Moreover, the doping induced PL suppression manifests itself more strongly for the low-energy than for the high-energy PL peaks. At $x=0.15$ only the high-energy peak located at $1.28 \mathrm{eV}(T=10 \mathrm{~K})$ survives that allows us to assume that the STE decay becomes more effective with doping. The high-energy PL peak red-shifts with the lowering the temperature, and its intensity decreases.

All these features can be consistently explained in frames of the STE nature of PL. Different PL peak can be assigned both to different STE and its clusters pointing to the multistage character of the luminescence.

Cuprates are believed to be unconventional systems which are unstable with regard to a self-trapping of the low-energy charge transfer excitons with a nucleation of electron-hole (EH) droplets being actually the system of coupled electron $\mathrm{CuO}_{4}^{7-}$ and hole $\mathrm{CuO}_{4}^{5-}$ centers having been glued in lattice due to a strong electron-lattice polarization effects. Phase transition to novel hypothetically metallic state could be realized due to a mechanism familiar to semiconductors with filled bands such as Ge and Si where given certain conditions one observes a formation of metallic EH-liquid as a result of the exciton decay 33 The system of strongly correlated electron $\mathrm{CuO}_{4}^{7-}$ and hole $\mathrm{CuO}_{4}^{5-}$ centers appears to be equivalent to an electron-hole Bose-liquid (EHBL) in contrast with the electron-hole Fermi-liquid in conventional semiconductors. A simple model description of such a liquid implies a system of local singlet bosons with a charge of $q=2 e$ moving in a lattice formed by hole centers. Local 
boson in our scenario represents the electron counterpart of Zhang-Rice singlet, or two-electron configuration $b_{1 g}^{2}{ }^{1} A_{1 g}$. Naturally, that conventional electron $\mathrm{CuO}_{4}^{7-}$ center represents a relaxed state of composite system: "hole $\mathrm{CuO}_{4}^{5-}$ center plus local singlet boson", while the "non-retarded" scenario of a novel phase is assumed to incorporate the unconventional states of electron $\mathrm{CuO}_{4}^{7-}$ center up to its orbital degeneracy.

Thus we can introduce the concept of insulator-toEHBL transition as the spontaneous condensation of selftrapped excitons and its clusters. However, in cuprates we deal with the electron/hole injection to the insulating parent phase due to a nonisovalent substitution as in $\mathrm{La}_{2-x} \mathrm{Sr}_{x} \mathrm{CuO}_{4}, \mathrm{Nd}_{2-x} \mathrm{Ce}_{x} \mathrm{CuO}_{4}$, or change in oxygen stoihiometry as in $\mathrm{YBa}_{2} \mathrm{Cu}_{3} \mathrm{O}_{6+x}, \mathrm{La}_{2} \mathrm{CuO}_{4-\delta}$, $\mathrm{La}_{2} \mathrm{Cu}_{1-x} \mathrm{Li}_{x} \mathrm{O}_{4}$. Such a substitution provokes the nucleation of $\mathrm{EH}$ droplets and shifts the phase equilibrium from the insulating state to the unconventional electron-hole Bose liquid, or, in other words, induces the insulator-to-EHBL phase transition. Hence, the formation of EHBL in cuprates can be considered as the first order phase transition. The doping in cuprates gradually shifts the EHBL state away from half-filing.

It is clear that the EHBL scenario makes the doped cuprates the objects of bosonic physics. There are numerous experimental evidence that support the bosonic

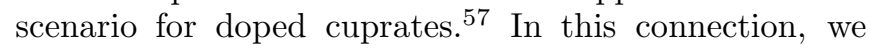
would like to draw attention to the little known results of comparative high-temperature studies of thermoelectric power and conductivity which unambiguously reveal the charge carriers with $q=2 e$, or two-electron(hole) transport. 58 The well-known relation $\frac{\partial \alpha}{\partial \ln \sigma}=$ const $=$ $-\frac{k}{q}$ with $|q|=2|e|$ is fulfilled with high accuracy in the limit of high temperatures $(\sim 700 \div 1000 K)$ for different cuprates $\left(\mathrm{YBa}_{2} \mathrm{Cu}_{3} \mathrm{O}_{6+x}, \mathrm{La}_{3} \mathrm{Ba}_{3} \mathrm{Cu}_{6} \mathrm{O}_{14+x}\right.$, $\left.\left(\mathrm{Nd}_{2 / 3} \mathrm{Ce}_{1 / 3}\right)_{4}\left(\mathrm{Ba}_{2 / 3} \mathrm{Nd}_{1 / 3}\right)_{4} \mathrm{Cu}_{6} \mathrm{O}_{16+x}\right)$.

\section{B. Manganites}

Parent manganites such as $\mathrm{LaMnO}_{3}$ are antiferromagnetic insulators with the charge transfer gap. Fundamental absorption band in parent manganites is formed both by the intracenter $\mathrm{O} 2 \mathrm{p}-\mathrm{Mn} 3 \mathrm{~d}$ transfer ${ }^{59}$ and by the small intercenter charge transfer excitons,, 60 which in terms of chemical notions represent somewhat like the disproportionation quanta with a rather low threshold of the order of $3 \mathrm{eV}$, resulting in a formation of electron $\mathrm{MnO}_{6}^{10-}$ and hole $\mathrm{MnO}_{6}^{8-}$ centers. The CT excitons in $\mathrm{LaMnO}_{3}$ prone to a self-trapping 61 and may be considered to be well defined entities only at small content, whereas at large densities their coupling is screened and their overlap becomes so considerable that they loose individuality, become unstable with regard to the decay (the dissociation) to electron and hole centers, and we come to a system of electrons and holes, which form an EH Bose liquid.

An instability of parent manganite $\mathrm{LaMnO}_{3}$ with re- gard to the overall disproportionation such as

$$
\mathrm{MnO}_{6}^{9-}+\mathrm{MnO}_{6}^{9-} \rightarrow \mathrm{MnO}_{6}^{10-}+\mathrm{MnO}_{6}^{8-}
$$

was strikingly demonstrated recently by Zhou and Goodenough ${ }^{62}$ The transport (thermoelectric power and resistivity) and magnetic (susceptibility) measurements showed that $\mathrm{LaMnO}_{3}$ above the cooperative Jahn-Teller orbital-ordering temperature $T_{J T} \approx 750 \mathrm{~K}$ transforms into charge-disproportionated paramagnetic phase with $\mu_{\text {eff }}=5.22 \mu_{B}$ and cooperative charge transfer of many heavy vibronic charge carriers. It seems rather obvious that with the lowering the temperature we arrive at a system with the well developed fluctuations of the $\mathrm{EH}$ Bose-liquid phase. Strong variation of the $\mathrm{LaMnO}_{3} \mathrm{Ra}-$ man spectra with increasing laser power 63 could be related to the photo-induced nucleation and the volume expansion of the EH Bose-liquid, especially, as at a rather big excitation wavelength $\lambda=514.5 \mathrm{~nm}$, at $\lambda=632.8 \mathrm{~nm}$ the absorption is considerably stronger in the domains of novel phase than in parent lattice.

Effective nucleation of the EH Bose-liquid in manganites could be provoked by a non-isovalent substitution since this strongly polarizable or even metallic phase in contrast with parent insulating phase provides an effective screening of charge inhomogeneity. Indeed, in thermoelectric power (TEP) experiments with doped manganites such as $\mathrm{La}_{1-x} \mathrm{Sr}_{x} \mathrm{MnO}_{3}$ Hundley and Neumeier 64 have found that more hole-like charge carriers or alternatively fewer accessible Mn sites are present than expected for the value $x$. They suggest a charge disproportionation model based on the instability of $\mathrm{Mn}^{3+}-\mathrm{Mn}^{3+}$ relative to that of $\mathrm{Mn}^{4+}-\mathrm{Mn}^{2+}$. This transformation provides excellent agreement with doping-dependent trends exhibited by both TEP and resistivity.

A simplified "chemical" approach to an EH Bose-liquid as to a disproportionated phase ${ }^{14}$ naively implies an occurrence of $\mathrm{Mn}^{4+}$ and $\mathrm{Mn}^{2+}$ ions. However, such an approach is very far from reality. Indeed, the electron $\mathrm{MnO}_{6}^{10-}$ and hole $\mathrm{MnO}_{6}^{8-}$ centers are already the mixed valence centers,, 65 as in the former the $M n$ valence resonates between +2 and +1 , and in the latter does between +4 and +3 .

In this connection, one should note that in a sense disproportionation reaction (46) has several purely ionic counterparts, the two rather simple

$$
\begin{aligned}
& \mathrm{Mn}^{3+}-\mathrm{O}^{2-}-\mathrm{Mn}^{3+} \rightarrow \mathrm{Mn}^{2+}-\mathrm{O}^{2-}-\mathrm{Mn}^{4+}, \\
& \text { and } \\
& \mathrm{Mn}^{3+}-\mathrm{O}^{2-}-\mathrm{Mn}^{3+} \rightarrow \mathrm{Mn}^{2+}-\mathrm{O}^{1-}-\mathrm{Mn}^{3+}, \\
& \text { and, finally, one rather complicated } \\
& \mathrm{O}^{2-}-\mathrm{Mn}^{3+} \mathrm{O}^{2-} \rightarrow \mathrm{O}^{1-}-\mathrm{Mn}^{1+} \mathrm{O}^{1-} \text {. }
\end{aligned}
$$

Thus, the disproportionation (46) threshold energy has to be maximally close to the CT energy parameter $\Delta_{p d}$. Moreover, namely this is seemingly to be one of the main parameters governing the nucleation of EH Bose-liquid in oxides.

So far, there has been no systematic exploration of exact valence and spin state of $\mathrm{Mn}$ in these systems. Park et $a l .66$ attempted to support the $\mathrm{Mn}^{3+} / \mathrm{Mn}^{4+}$ model, based 
on the Mn 2p x-ray photoelectron spectroscopy (XPES) and O1s absorption. However, the significant discrepancy between the weighted $\mathrm{Mn}^{3+} / \mathrm{Mn}^{4+}$ spectrum and the experimental one for given $x$ suggests a more complex doping effect. Subias et al ${ }^{67}$ examined the valence state of Mn utilizing Mn $K$-edge x-ray absorption near edge spectra (XANES), however, a large discrepancy is found between experimental spectra given intermediate doping and appropriate superposition of the end members.

The valence state of $\mathrm{Mn}$ in Ca-doped $\mathrm{LaMnO}_{3}$ was studied by high-resolution Mn $K \beta$ emission spectroscopy by Tyson et al ${ }^{68}$. No evidence for $\mathrm{Mn}^{2+}$ was observed at any $x$ values seemingly ruling out proposals regarding the $\mathrm{Mn}^{3+}$ disproportionation. However, this conclusion seems to be absolutely unreasonable. Indeed, electron center $\mathrm{MnO}_{6}^{10-}$ can be found in two configuration with formal $\mathrm{Mn}$ valence $\mathrm{Mn}^{2+}$ and $\mathrm{Mn}^{1+}$ (not simply $\mathrm{Mn}^{2+}$ !), respectively. In its turn, the hole center $\mathrm{MnO}_{6}^{8-}$ can be found in two configurations with formal Mn valence $\mathrm{Mn}^{4+}$ and $\mathrm{Mn}^{3+}$ (not simply $\mathrm{Mn}^{4+}$ !), respectively. So, within the model the Mn $K \beta$ emission spectrum for Ca-doped $\mathrm{LaMnO}_{3}$ has to be a superposition of appropriately weighted $\mathrm{Mn}^{1+}, \mathrm{Mn}^{2+}, \mathrm{Mn}^{3+}, \mathrm{Mn}^{4+}$ contributions (not simply $\mathrm{Mn}^{4+}$ and $\mathrm{Mn}^{3+}$, as one assumes in Ref. 68). Unfortunately, we do not know the Mn $K \beta$ emission spectra for the oxide compounds with $\mathrm{Mn}^{1+}$ ions, however a close inspection of the $\mathrm{Mn} K \beta$ emission spectra for the series of Mn-oxide compounds with $\mathrm{Mn}$ valence varying from $2+$ to $7+$ (Fig.2 of the cited paper) allows to uncover a rather clear dependence on valence, and indicates a possibility to explain the experimental spectrum for Ca-doped $\mathrm{LaMnO}_{3}$ as a superposition of appropriately weighted $\mathrm{Mn}^{1+}, \mathrm{Mn}^{2+}, \mathrm{Mn}^{3+}, \mathrm{Mn}^{4+}$ contributions. It should be noted that an "arrested" Mn-valence response to the doping in the $x<0.3$ range founded in Ref. 68 is also consistent with the creation of predominantly oxygen holes.

This set of conflicting data together with a number of additional data ${ }^{69}$ suggests the need for an in-depth exploration of the $\mathrm{Mn}$ valence problem in this perovskite system. However, one might say, the doped manganites are not only systems with mixed valence, but systems with indefinite valence, where we cannot, strictly speaking, unambiguously distinguish Mn species with either distinct valence state.

\section{CONCLUSIONS}

We have developed a model approach to describe charge fluctuations and different charge phases in strongly correlated $3 \mathrm{~d}$ oxides. In frames of $S=1$ pseudospin formalism different phase states of the system of the metal-oxide $M$ centers with three different valent state $M^{0, \pm}$ are considered on the equal footing. Simple uniform mean-field phases include an insulating monovalent $M^{0}$-phase, mixed-valence binary (disproportionated) $M^{ \pm}$-phase, and mixed-valence ternary ("underdisproportionated") $M^{0, \pm}$-phase. We consider two first phases in more details focusing on the problem of electron/hole states and different types of excitons in $M^{0}$ phase and formation of electron-hole Bose liquid in $M^{ \pm}$. phase.

Our consideration was focused mainly on a number of issues seemingly being of primary importance for the various strongly correlated oxides such as cuprates, manganites, bismuthates, and other systems with CT instability and/or mixed valence. These includes two types of single particle correlated hopping and the two-particle hopping, CT excitons, electron-lattice polarization effects which are shown to be crucial for the stabilization of either phase, topological charge fluctuations, nucleation of droplets of the electron-hole Bose liquid and phase separation effect. We emphasize an important role of selftrapped CT excitons in typical Mott-Hubbard insulators as candidate "relaxed excited states" to struggle for stability with ground state and natural nucleation centers for unconventional electron-hole Bose liquid which phase state include the superfluid.

Pseudo-spin formalism has appeared to be very efficient to reveal and describe different aspects of essential physics for mixed-valence system. We show that the coherent states provide the optimal way both to a correct mean-field approximation and respective continuous models to describe the pseudo-spin system including different topological charge fluctuations, in particular, like domain walls or bubble domains in antiferromagnets. All the insulating systems such as $M^{0}$-phase may be subdivided to two classes: stable and unstable ones with regard to the formation of self-trapped CT excitons. The latter systems appear to be unstable with regard the formation of CT exciton clusters, or droplets of the electron-hole Bose liquid. The model approach suggested is believed to provide a conceptual framework for an in-depth understanding of physics of strongly correlated oxides such as cuprates, manganites, bismuthates, and other systems with charge transfer excitonic instability and/or mixed valence. We shortly discuss an unconventional scenario of the essential physics of cuprates and manganites that implies their instability with regard to the self-trapping of charge transfer excitons and the formation of electronhole Bose liquid.

Author acknowledges the stimulating discussions with V. Vikhnin, A.V. Mitin, S.-L. Drechsler, T. Mishonov, R. Hayn, I. Eremin, M. Eremin, Yu. Panov, V.L. Kozhevnikov and support by SMWK Grant, INTAS Grant No. 01-0654, CRDF Grant No. REC-005, RFBR Grant No. 04-02-96077. 
1 J. G. Bednorz and K. A. Müller, Z. Phys. B, 4, 189 (1986).

2 M. Imada et. al., Rev. Mod. Phys. 70, 1039 (1998).

3 N.F. Mott, Metal-Insulator Transitions, 2nd ed (Taylor\&Francis, London, 1990).

4 A. L. Shluger and A. M. Stoneham, J. Phys.: Condens. Matter 53049 (1993).

5 V.S. Vikhnin, S. Avanesyan, H. Liu, S.E. Kapphan, J. Phys. Chem. Solids, 63, 1677 (2002).

6 J.E. Hirsch and S. Tang, Phys. Rev. B 40, 2179 (1989); J.E. Hirsch, in "Polarons and bipolarons in high- $T_{c}$ superconductors and related materials", eds E.K.H. Salje, A.S. Alexandrov and W.Y. Liang, Cambridge University Press, 1995, p. 234.

7 A.S. Moskvin and Yu.D. Panov, Phys. Rev. B 68, 125109 (2003).

8 P. Huai and Keiichiro Nasu, J. Phys. Soc. Jap. 71, 1182 (2002).

9 W.P. Su, J.R. Schrieffer, A.J. Heeger, Phys. Rev. B 22, 2099 (1980).

10 Y. Ando, A.N. Lavrov, S. Komiya, K. Segawa, and X.F. Sun, Phys. Rev. Lett. 87, 017001 (2001).

11 Relaxations of Excited States and Photo-Induced Structural Phase Transitions, ed.: K. Nasu, Springer Series in SolidState Sciences, V. 124, p.17 (1997).

12 M.Yu. Kagan, K.I. Kugel, and D.I. Khomskii, JETP, 93, 415 (2001).

13 T. Mizokawa, D.I. Khomskii, and G.A. Sawatzky, Phys. Rev., B61, 11263 (2000).

14 S.P. Ionov, G.V. Ionova, V.S. Lubimov, E.F. Makarov, Phys. Stat. Sol. (b), 71, 11 (1975).

15 A.S. Moskvin, The Physics of Metals and Metallography Vol. 95, Suppl. 1, 2003, p. 41

16 H. Eskes, L.H. Tjeng and G.A. Sawatzky, Phys. Rev., B 41, 288 (1990).

17 J. Ghijsen, L.H. Tjeng, J. van Elp et. al., Phys. Rev., B 38, 11322 (1988).

18 A.S. Moskvin, R. Neudert, M. Knupfer, J. Fink, and R. Hayn, Phys. Rev. B 65, 180512(R) (2002).

19 Zhang F.C. and Rice T.M., Phys.Rev, B37, 3759 (1988).

20 M.S. Hybertsen, M. Schluter, N.E. Christensen, Phys. Rev. B B 39, 9028 (1989).

21 M.S. Hybertsen, E.B. Stechel, M. Schluter, D.R. Jennison, Phys. Rev., B 41, 11068 (1990).

22 R. Nataf, Les modeles en spectroscopie nucleaire, Dunod, Paris, 1965.

23 J.E. Hirsch, Phys. Rev. Lett. 87, 206402 (2001); Phys.Rev. B 65, 184502 (2002).

24 A.S. Moskvin, V.A. Korotaev, Yu.D. Panov, M.A. Sidorov, Physica C, 282-287, 1735 (1997).

25 Yu.D. Panov, A.S. Moskvin, Phys. Solid State, 42, 846 (2000).

26 B.G. Dick and A.W. Overhauser, Phys. Rev. 112, 90 (1958).

27 W. Kauzman, Quantum Chemistry (Academic, New York, 1957).

28 I.B. Bersuker and V.Z. Polinger, Vibronic Interactions in Molecules and Crystals, (Springer-Verlag, Berlin, 1989).

29 D. A. Varshalovich, A. N. Moskalev, V. K. Khersonskii. Quantum Theory of Angular Momentum (World Scientific, Singapore, 1988).

30 N.A. Mikushina, A.S. Moskvin, Phys. Lett. A 302, 8
(2002); arXiv:cond-mat/0111201

31 M. Boman, R. J. Bursill, Phys. Rev., B 57, 15167 (1998).

32 D. Guo, S. Mazumdar, S.N. Dixit et. al., Phys. Rev., B 48, 1433 (1993-I).

33 T.M. Rice, in Solid State Physics, Eds.H. Ehrenreich, F. Seitz, D. Turnbull, 32, 1 (1977).

34 A.S. Moskvin, J. Phys.: Conference Ser. (to be published)

35 A. I. Kirilyuk, N. M. Kreines, and V. I. Kudinov, Pisma Zh. Eksp. Teor. Fiz. 52, 696 (1990) [JETP Lett. 52, 49 (1990)].

36 R. Micnas, J. Ranninger and S. Robaszkiewicz, Rev. Mod. Phys. 62, 113 (1990).

37 R.T. Scalettar, G.G. Batrouni, A.P. Kampf, and G.T. Zimanyi, Phys. Rev. B 51, 8467 (1995-I).

38 K. Bernardet, G.G. Batrouni, J.-L. Meunier et. al., Phys. Rev. B 65, 104519 (2002).

39 A.A. Belavin, A.M. Polyakov, JETP Lett. 22 (1975) 245.

40 O. Penrose and L. Onsager, Phys. Rev. 104, 576 (1956).

41 G. G. Batrouni and R. T. Scalettar, Phys. Rev. Lett. 84, 1599 (2000).

42 F. Hébert, G.G. Batrouni, R.T. Scalettar et. al., Phys. Rev. B 65, 014513 (2001).

43 Guido Schmid, Synge Todo, Matthias Troyer, and Ansgar Dorneich, Phys. Rev. Lett. 88, 167208-1 (2002).

44 A.S. Moskvin, I.G. Bostrem, A.S. Ovchinnikov, JETP Lett. 78, 772 (2003).

45 A. S. Moskvin, Phys. Rev. B 69, 214505 (2004).

46 A.S. Moskvin, Yu.D. Panov, N.V. Mel'nikova, JETP, 99, 1108 (2004).

47 S.A. Kivelson, B.Z. Spivak, Phys. Rev. B 45, 10490 (1992).

48 A.G. Green, Phys. Rev. B 61, R16299 (2000).

49 Carsten Timm, S.M. Girvin, H.A. Fertig, Phys. Rev. B 58, 10634 (1998).

50 A. S. Moskvin, J. Malek, M. Knupfer, R. Neudert, J. Fink, R. Hayn, S.-L. Drechsler, N. Motoyama, H. Eisaki, and S. Uchida, Phys. Rev. Lett. 91, 037001 (2003).

51 J.B. Goodenough, J. Supercond. 13, 793 (2000).

52 D. Salamon, Ran Liu, M.V. Klein et al., Phys. Rev. B 51, 6617 (1995-II).

53 J.M. Ginder, M. G. Roe, Y. Song et. al., Phys. Rev. B 37, 7506 (1988).

54 V. N. Denisov, C. Taliani, A.G. Mal'shukov et. al., Phys. Rev. B 48, 16714 (1993-II).

55 V.V. Eremenko, T.V. Sukhareva, and V.N. Samovarov, Phys. Solid State, 42, 816 (2002).

56 S. Sugai, M. Sato, T. Kobayashi et al., Phys. Rev. B 42, 1045 (1990).

57 A.S. Alexandrov and N.F. Mott, J. Superconductivity, 7, 599 (1994); A.S. Alexandrov, Physica C 305, 46 (1998).

58 I.A. Leonidov, Y.N. Blinovskov, E.E. Flyatau, P.Y. Novak, V.L. Kozhevnikov, Physica C, 158, 287 (1989); M.-Y. Su, C.E. Elsbernd, T.O. Mason, J. Amer. Cer. Soc., 73, 415 (1990); E.B. Mitberg, M.V. Patrakeev, A.A. Lakhtin et al., J. Alloys and Compounds, 274, 103 (1998).

59 A.S. Moskvin, Phys. Rev. B 65, 205113 (2002).

60 N.N. Kovaleva et al., Phys. Rev. Lett. 93, 147204 (2004).

61 N. N. Kovaleva, G.L. Gavartin, A. L. Shluger et al., JETP, 94, 178 (2002).

62 J.-S. Zhou and J.B. Goodenough, Phys. Rev. B 60, R15002 (1999).

63 M.N. Iliev, M.V. Abrashev, H.-G. Lee et al., Phys. Rev. B 
57, $2872(1998)$.

64 M.F. Hundley, J.J. Neumeier, Phys. Rev. B 55, 11511 (1997).

65 A.S. Moskvin, I.L. Avvakumov, Physica B, 322/3-4, 371 (2002); arXiv:cond-mat/0108355

66 J.-H. Park, C.T. Chen, S.-W. Cheong et al., Phys. Rev. Lett. 76, 4215 (1999).

67 G. Subias, J. Garcia, M.G. Proietti et al., Phys. Rev. B
56, 8183 (1997).

68 T.A. Tyson, Q. Qian, C.-C. Kao et al., Phys. Rev. B 60, 4665 (1999).

69 M. Croft, D. Sills, M. Greenblatt et al., Phys. Rev. B 55, 8726 (1997); R.S. Liu, J.B. Wu, C.Y. Chang et al., J. Sol. St. Chem. 125, 112 (1996). 